AEI-2002-040

CERN-TH/2002-115

LAPTH-915/02

hep-th/0205270

\title{
Conformal fields in the pp-wave limit
}

\author{
G. Arutyunov,**円，E. Sokatchev ${ }^{\ddagger, \neq \ddagger}$ 月 \\ * Max-Planck-Institut für Gravitationsphysik, Albert-Einstein-Institut, \\ Am Mühlenberg 1, D-14476 Golm, Germany \\ ‡ CERN Theoretical Division, CH12-11 Geneva 23, Switzerland
}

\begin{abstract}
The pp-wave (Penrose limit) in conformal field theory can be viewed as a special contraction of the unitary representations of the conformal group. We study the kinematics of conformal fields in this limit in a geometric approach where the effect of the contraction can be visualized as an expansion of space-time. We discuss the two common models of space-time as carrier spaces for conformal fields: One is the usual Minkowski space and the other is the coset of the conformal group over its maximal compact subgroup. We show that only the latter manifold and the corresponding conformal representation theory admit a non-singular contraction limit. We also address the issue of correlation functions of conformal fields in the pp-wave limit. We show that they have a well-defined contraction limit if their space-time dependence merges with the dependence on the coordinates of the $\mathrm{R}$ symmetry group. This is a manifestation of the fact that in the limit the space-time and $\mathrm{R}$ symmetries become indistinguishable. Our results might find applications in actual calculations of correlation functions of composite operators in $\mathcal{N}=4$ super Yang-Mills theory.
\end{abstract}

\footnotetext{
${ }^{1}$ email:agleb@aei-potsdam.mpg.de

2 email:Emery. Sokatchev@cern.ch

** On leave of absence from Steklov Mathematical Institute, Gubkin str.8, 117966, Moscow, Russia

${ }^{\ddagger \ddagger}$ On leave of absence from Laboratoire d'Annecy-le-Vieux de Physique Théorique LAPTH, B.P. 110, F74941 Annecy-le-Vieux et l'Université de Savoie
} 


\section{Introduction}

$\mathcal{N}=4$ super Yang-Mills theory is a non-trivial interacting superconformal field theory which is believed to possess a holographic (dual) description in terms of type IIB string theory on an $A d S_{5} \times S^{5}$ background [1]. Apart from $A d S_{5} \times S^{5}$ and flat Minkowski space, there exists another maximally supersymmetric background referred to as the "pp-wave solution" [2]. This background also arises as the Penrose limit of the $A d S_{5} \times S^{5}$ space [3]. Thus, it is of obvious interest to understand what happens to the AdS/CFT duality in the Penrose limit.

One of the nice features of the pp-wave solution is that the spectrum of a free string

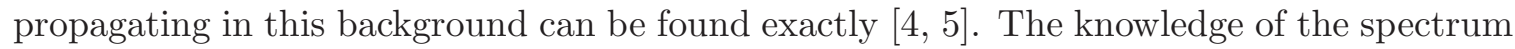
offers an opportunity to study the duality conjecture beyond the supergravity approximation. Recently, an interesting proposal [6] has been made to identify certain classes of gauge invariant composite operators in $\mathcal{N}=4$ theory with states of the supergravity and string theory in the pp-wave background.

Both the kinematical and dynamical information about any gauge theory is encoded in the correlation functions of gauge invariant composite operators. Such operators form unitary irreducible representations (UIRs) of the superconformal algebra $\operatorname{PSU}(2,2 \mid 4)$ and the lowest bosonic components of the corresponding supermultiplets are specified by their conformal dimension $\Delta$, the two Lorentz spins of $\mathrm{SO}(3,1)$ and by a finite-dimensional UIR of the R symmetry group SU(4). On the gauge theory side the Penrose limit consists in taking the rank $N$ of the gauge group $\mathrm{SU}(N)$ to infinity, while keeping the Yang-Mills coupling constant $g$ fixed. According to the proposal of Ref. [6], the operators surviving in this limit have a very large conformal dimension $\Delta \sim \sqrt{N}$ and a very large $\mathrm{U}(1)$ charge $J$ (where $\mathrm{U}(1)$ is a subgroup of the $\mathrm{R}$ symmetry group), such that the ratio $J^{2} / N$ and the difference $\Delta-J$ are kept finite. Some interesting perturbative computations of two- and three-point correlation functions in $\mathcal{N}=4$ theory have been done recently [7, 8, 9] and they provide further evidence that the well-defined perturbative parameter of the gauge theory in this limit is $g^{2} N / J^{2}$.

However, we feel that the present understanding of the basic principles of the conformal field theory (CFT) in this limit is not yet complete. Above all, this concerns the precise definition of the Hilbert space of states and of the correlation functions. Indeed, the operators we are interested in have infinite canonical dimension and therefore the space-time dependence of their correlation functions requires a special treatment. In particular, one would like to understand the space-time manifold where the dual gauge theory lives. Some of these questions have already been addressed in the current literature [10, 11, 12].

The pp-wave limit can be viewed as the passage from the superconformal algebra $\operatorname{PSU}(2,2 \mid 4)$ to the symmetry superalgebra of the pp-wave background. The bosonic part of the latter is the semi-direct sum of $\mathrm{h}(8)$ with the external automorphism algebra $\mathrm{so}(4) \oplus \mathrm{so}(4)$ $\oplus$ so(2). Here $h(8)$ denotes two copies of the four-dimensional Heisenberg algebra $h(4)$ with a common central element. This passage is just a generalized Inönü-Wigner contraction [2, 13]. The contraction parameter is related to the infinite conformal dimension of the fields.

The conformal fields are UIRs of the conformal group. Therefore, one can try to carry out the contraction procedure directly in the Hilbert space of conformal fields in order to see which states survive in the contraction limit and give rise to the Hilbert space of states of the contracted group. Studying conformal kinematics in the pp-wave limit is the aim of 
the present paper.

The main problem that one is confronted with is the infinite conformal dimension $\Delta$ of the fields. We propose a mechanism in which this infinite weight is compensated by another infinite quantum number carried by the fields, a charge $J$ coming from the R symmetry sector. Traditionally, the conformal fields $\varphi\left(x^{\mu}\right)$ posses a definite dilatation weight, just like the Minkowski space coordinates $x^{\mu}$. This dilatation weight is the representation label for the non-compact subgroup $\mathrm{SO}(1,1) \sim \mathbb{R}$ of the conformal group $\mathrm{SO}(4,2)$. However, the compact R symmetry group $\mathrm{SO}(6)$ does not have such a subgroup, so the compensation can only take place between two $\mathrm{SO}(2)$ subgroups of $\mathrm{SO}(4,2)$ and of $\mathrm{SO}(6)$. The group $\mathrm{SO}(2)$ is part of the maximal compact subgroup $\mathrm{K}=\mathrm{SO}(4) \times \mathrm{SO}(2)$ of the conformal group $\mathrm{G}=$ $\mathrm{SO}(4,2)$. Thus, it is natural to introduce conformal fields in such a way that they form a linear representation of the maximal compact subgroup $\mathrm{K}$.

In general, the spaces in which the conformal fields are defined can be regarded as coset spaces $H \backslash G$ corresponding to different choices of the subgroup $H \subset G$. A formulation with a linear action of $K$ should be based on the coset space $\mathbb{H}_{+}^{4}=K \backslash G$. This coset is a fourdimensional non-compact Kähler manifold which can be viewed as a bounded domain in $\mathbb{C}^{4}$. The free conformal fields correspond to the so-called discrete series of UIRs of G. They can be realized as functions, analytic in $\mathbb{H}_{+}^{4}$ and transforming homogeneously and irreducibly in finite-dimensional UIRs of K. One says that such UIRs of G are "induced" by the compact subgroup K.

Apart from the conformal group, the fields of the $\mathcal{N}=4$ SYM theory are also UIRs of the $\mathrm{R}$ symmetry group. It is then natural (and even necessary in the contraction context, as we argue) to realize this internal symmetry in an auxiliary compact space. It is obtained by dividing the $\mathrm{R}$ symmetry group $\mathrm{SO}(6)$ by a maximal compact subgroup, which we choose to be the same $\mathrm{K}=\mathrm{SO}(4) \times \mathrm{SO}(2)$. Only then, by examining the combined action of the two $\mathrm{SO}(2)$ subgroups from the conformal and $\mathrm{R}$ symmetry sectors, we are able to define a contraction limit in the Hilbert space of the conformal fields, such that one of the two groups $\mathrm{SO}(2)$ survives. In this way we obtain the Bargmann-Fock representation of the Heisenberg algebra, in which the generator of the surviving $\mathrm{SO}(2)$ plays the rôle of the Hamiltonian. In the process of contraction the bounded domain $\mathbb{H}_{+}^{4}$ is expanded to the full space $\mathbb{C}^{4}$.

This picture is quite different from the traditional approach to conformal fields in real (compactified) Minkowski space. There the UIRs of $\mathrm{G}$ are induced by its parabolic subgroup consisting of the Lorentz group $\mathrm{SO}(1,3)$, dilatations $\mathrm{SO}(1,1)$ and the Abelian subgroup of conformal boosts. The resulting space $\overline{\mathbb{M}}^{4}=\left(S^{1} \times S^{3}\right) / \mathbb{Z}_{2}$ can be viewed as the boundary of the non-compact space $\mathbb{H}_{+}^{4}$. Consequently, the conformal fields in $\overline{\mathbb{M}}^{4}$ are in fact the boundary values of the analytic functions in $\mathbb{H}_{+}^{4}$. In particular, one can show that the two spaces provide two different but equivalent realizations of the discrete series of UIRs of $G$. Note that $\mathrm{K}$ acts as an isometry of $\overline{\mathbb{M}}^{4}$ and one can label the conformal fields on $\overline{\mathbb{M}}^{4}$ by the quantum numbers of K. The correlation functions of the conformal fields on $\overline{\mathbb{M}}^{4}$ (the Wightman functions) are also the limiting values of analytic functions on $\mathbb{H}_{+}^{4}$.

Since in the contraction limit the bounded domain $\mathbb{H}_{+}^{4}$ blows up into $\mathbb{C}^{4}$, it looses its boundary and, as a consequence, the relationship with the representation theory in $\overline{\mathbb{M}}^{4}$ apparently breaks down. This clearly shows that the contraction, viewed by a Minkowski space observer, is a singular process. At present we are not able to exhibit a well-defined contraction limit for conformal fields initially defined on $\overline{\mathbb{M}}^{4}$. This problem requires further 
study.

All the main features of the contraction procedure described above are already seen in the simplest one-dimensional case. There the symmetry group is $\mathrm{SO}(1,2) \times \mathrm{SO}(3) \sim$ $\mathrm{SU}(1,1) \times \mathrm{SU}(2)$. The bounded complex domain is the upper sheet of the double-sheeted hyperboloid $\mathbb{H}_{+}^{1} \sim \mathrm{SO}(2) \backslash \mathrm{SO}(1,2)$ (which is also isomorphic to the Poincaré disc $\mathcal{D}^{1}$ ). The internal space is the two-sphere $S^{2} \sim \mathrm{SO}(2) \backslash \mathrm{SO}(3)$. For the sake of simplicity, we restrict the discussion in this paper to the one-dimensional case.

The paper is organized as follows. In Section 2 we briefly describe the realizations of the bosonic algebra so $(1,2) \oplus \mathrm{so}(3)$ in different coset spaces. In particular, we recall Dirac's manifestly covariant realization of the conformal group on the light cone. It serves as a good introduction to the approach to fields on coset spaces $K \backslash G$ that we adopt: We regard them as functions on the group $G$ homogeneous under the left action of the subgroup K. Section 3 contains some fairly standard material [14, 15] about the UIRs of the groups $\operatorname{SO}(1,2)$ and $\mathrm{SO}(3)$. In particular, we explain how the discrete series of UIRs of $\mathrm{SO}(1,2)$ (which correspond to free conformal fields) can be constructed in a spinor basis. Then we derive the matrix elements of these representations which are to be used as an orthogonal basis for expanding conformal fields. Choosing to diagonalize either the $\mathrm{SO}(2)$ or $\mathrm{SO}(1,1)$ subgroups of $\mathrm{SO}(1,2)$ leads to different notions of conformal fields, carrying either a compact charge or a dilatation weight, respectively. Only the former turns out suitable for the pp-wave limit. In Section 4 we show how the free conformal fields living on the unit disc and on the two-sphere can be contracted in the pp-wave limit. The main point here is to expand the unit disc so that it covers the whole plane. In the process the conformal weight and the $\mathrm{R}$ charge grow as the square of the disc radius. We briefly discuss two possibilities to contract fields living in the compact Minkowski space (the circle $S^{1}$ in this case) and explain why they fail. Finally, in Section 5 we construct correlation functions of primary fields on $\mathcal{D}^{1} \times S^{2}$ and present a possible well-defined and non-trivial contraction limit. In view of the holographic duality it would be interesting to understand how these correlation functions could emerge from string theory.

\section{Generalities}

\subsection{The conformal and $R$ symmetry algebras}

The bosonic part of the symmetry group of the $\mathcal{N}=4$ SYM theory in four dimensions is $\mathrm{SO}(4,2) \times \mathrm{SO}(6)$, which is locally isomorphic to $\mathrm{SU}(2,2) \times \mathrm{SU}(4)$. All the main features of the pp-wave limit that we are discussing in this paper are perfectly well illustrated by the much simpler one-dimensional case. So, we shall restrict ourselves to one-dimensional conformal fields with symmetry group $\mathrm{SO}(1,2) \times \mathrm{SO}(3) \sim \mathrm{SU}(1,1) \times \mathrm{SU}(2)$.

The Lie algebra of $\mathrm{SO}(1,2) \sim \mathrm{SU}(1,1) \sim \mathrm{SL}(2, \mathbb{R})$ with antisymmetric generators $M_{a b}$, $M_{a b}=-M_{b a}$, has the following commutation relations

$$
\left[M_{a b}, M_{c d}\right]=\eta_{b c} M_{a d}+\eta_{a d} M_{b c}-\eta_{a c} M_{b d}-\eta_{b d} M_{a c},
$$

where $a, b=0,1,2$ and the metric is $\eta=\operatorname{diag}(-,+,-)$. The non-compact group $\mathrm{SO}(1,2)$ has two important one-dimensional subgroups, the non-compact $\mathrm{SO}(1,1)$ (generator $D \equiv M_{21}$ ) and the compact $\mathrm{SO}(2)$ (generator $M_{0} \equiv i M_{02}$ ). It is useful to rewrite the algebra (1) in two different bases with manifest covariance with respect to each of these subgroups. 
Introducing the notation $P=1 / \sqrt{2}\left(M_{02}-M_{01}\right)$ and $K=1 / \sqrt{2}\left(M_{01}+M_{02}\right)$, we can recast (1) in the form

$$
\begin{aligned}
& {[D, P]=-P,} \\
& {[D, K]=K,} \\
& {[P, K]=D .}
\end{aligned}
$$

Here $D, P$ and $K$ are the familiar generators of dilatations, (time) translations and conformal boosts, respectively.

The alternative basis is obtained by introducing complex combinations of generators, $M_{ \pm}=1 / \sqrt{2}\left(i M_{12} \mp M_{01}\right)$ :

$$
\begin{aligned}
& {\left[M_{0}, M_{ \pm}\right]= \pm M_{ \pm},} \\
& {\left[M_{+}, M_{-}\right]=-M_{0} .}
\end{aligned}
$$

Here the $\mathrm{SO}(2) \sim \mathrm{U}(1)$ generator $M_{0}$ and the raising $M_{+}$and lowering $M_{-}$operators have the following properties under Hermitian conjugation:

$$
M_{0}^{\dagger}=M_{0}, \quad M_{+}^{\dagger}=M_{-} .
$$

The representations of $\mathrm{SO}(1,2)$ are labeled by the quadratic Casimir

$$
C_{2}=D^{2}-P K-K P=M_{0}^{2}-M_{+} M_{-}-M_{-} M_{+} .
$$

The Lie algebra of $\mathrm{SO}(3) \sim \mathrm{SU}(2)$ with generators $L_{a b}, a, b=1,2,3$ has the same commutation relations (11), except that the metric now is $\eta_{a b}=\delta_{a b}$. This algebra admits a form analogous to (3), with the $\mathrm{SO}(2) \sim \mathrm{U}(1)$ generator $L_{0}=-i L_{12}$ and the raising and lowering operators $L_{ \pm}=i / \sqrt{2}\left(L_{12} \pm i L_{13}\right)$ :

$$
\begin{aligned}
& {\left[L_{0}, L_{ \pm}\right]= \pm L_{ \pm},} \\
& {\left[L_{+}, L_{-}\right]=L_{0},}
\end{aligned}
$$

with the same properties (田) under conjugation. The only difference between (6) and (3) is in the sign in front of the $\mathrm{U}(1)$ generator $L_{0}$ or $M_{0}$. The expression for the quadratic Casimir becomes

$$
C_{2}=L_{0}^{2}+L_{+} L_{-}+L_{-} L_{+}
$$

\subsection{Models of space-time}

Conformal fields can be introduced in several ways which are in correspondence with different group-theoretic decompositions of an element of the conformal group G. Below we list these possibilities for the one-dimensional case $\mathrm{G}=\mathrm{SO}(1,2)$.

\subsubsection{The compact space}

The first way to define a conformal field is based on the Bruhat decomposition $\mathrm{G}=$ NAMÑ valid for almost all elements of the group. H Here $\mathrm{N}$ is the nilpotent subgroup of special

\footnotetext{
${ }^{1}$ For more details see Section 3.4 .
} 

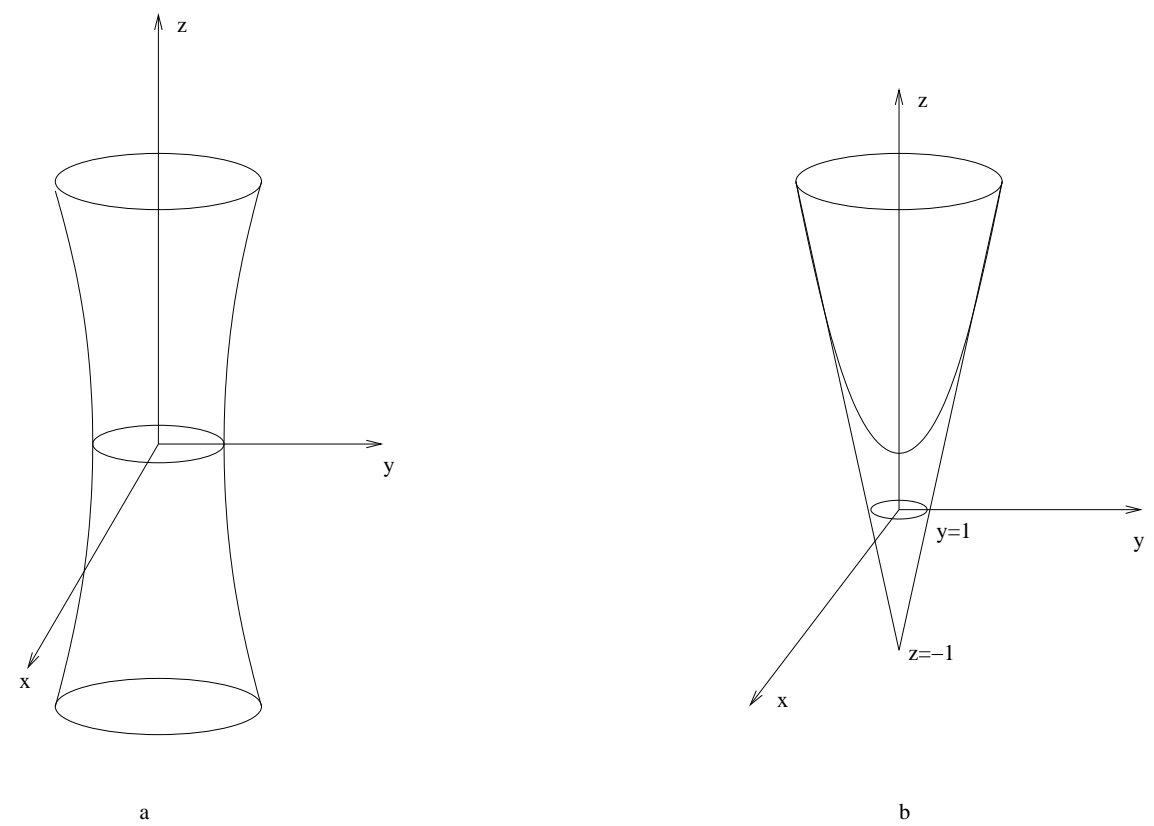

Figure 1: Models of the coset spaces $\mathbb{H}_{0}=\mathrm{SO}(1,1) \backslash \mathrm{SO}(1,2)$ and $\mathbb{H}_{+}=\mathrm{SO}(2) \backslash \mathrm{SO}(1,2)$. The Poincaré disc $\mathcal{D}^{1}$ arises as the projection of the upper sheet of the double-sheeted hyperboloid $\mathbb{H}_{+}$on the $x y$-plane. Regarded as a bounded complex domain, the disc is isomorphic to the coset space $\mathrm{U}(1) \backslash \mathrm{SU}(1,1)$.

conformal transformations ("boosts"), $\mathrm{M}$ is a center element (it becomes the Lorentz group in higher dimensions), $\mathrm{A}=\mathrm{SO}(1,1)$ is the group of dilatations and $\tilde{\mathrm{N}}$ the nilpotent subgroup of translations. The coset space $2 \mathrm{NM} \backslash \mathrm{G}$ is the positive cone $\mathrm{C}_{+}$in $\mathbb{R}^{1,2}$. Dividing further the cone by the dilatations we obtain the conformal compactification of the Minkowski space: $\overline{\mathbb{M}}^{1}=S^{1}=\mathrm{A} \backslash \mathrm{C}_{+}=\mathrm{ANM} \backslash \mathrm{G}$. Conformal fields are introduced as functions on $\mathrm{C}_{+}$ transforming homogeneously under A (see Section 2.3). Thus, effectively they are functions on the unit circle ANM $\backslash \mathrm{G}=S^{1}$. P In this construction the compact subgroup $\mathrm{K}=\mathrm{SO}(2)$ plays the rôle of the isometry group of the homogenous space $S^{1}$.

This coset space construction is depicted schematically in Figure 1a. The non-compact coset $\mathrm{SO}(1,1) \backslash \mathrm{SO}(1,2)$ is the single-sheeted hyperboloid. The further factorization by the nilpotent subgroup $\mathrm{N}$ corresponds to identifying the different sections (circles).

\subsubsection{The non-compact space}

The second way is based on the Iwasawa decomposition $\mathrm{G}=\mathrm{KAN}$, where $\mathrm{K}$ is the maximal compact subgroup. Dividing by $\mathrm{K}$, we introduce the non-compact coset space $\mathrm{K} \backslash \mathrm{G}=\mathrm{AN}$. The conformal fields are described as functions $f(g)$ on $\mathrm{G}$ obeying for any $k \in \mathrm{K}$ the covariance (homogeneity) condition $f(k g)=\rho(k) f(g)$, where $\rho$ is a finite-dimensional unitary

\footnotetext{
${ }^{2}$ The subgroup $\mathrm{N}$ is normal with respect to $\mathrm{A}$, therefore the order of the factors $\mathrm{N}$ and $\mathrm{A}$ can be changed.

${ }^{3}$ In physics it is customary to replace the compact manifold $S^{1}$ by the one-dimensional non-compact Minkowski space $\mathbb{M}^{1}=\mathbb{R}^{1}$. The compactification $\overline{\mathbb{M}}^{1}=S^{1}$ is recovered by completing the line $\mathbb{R}^{1}$ with the infinite point.
} 
irreducible representation (UIR) of $\mathrm{K}$.

In the one-dimensional case $\mathrm{K}=\mathrm{SO}(2)$ and the non-compact coset $\mathrm{SO}(2) \backslash \mathrm{SO}(1,2)$ is the upper sheet of the double-sheeted hyperboloid (Figure 1b). It can be equivalently represented by its stereographic projection onto the $x y$ plane, which is the open unit disc $\mathcal{D}^{1}$. The latter is also the image of the isomorphic coset space $\mathrm{U}(1) \backslash \mathrm{SU}(1,1)$.

The relation between the conformal spaces $S^{1}$ and $\mathcal{D}^{1}$ introduced above is established through the fact that the non-compact coset $\mathcal{D}^{1}$ is bounded and the boundary is precisely the compact Riemannian space $S^{1}$. The non-compact coset is a Kähler manifold with boundary. Holomorphic functions on this manifold are defined by their restriction to the boundary. This is how the conformal fields on two different cosets are related to each other.

It is important to realize that the above relationship between the compact and noncompact pictures persists in the four-dimensional case as well. There the compact Minkowski space is obtained by dividing the conformal group $\mathrm{SO}(4,2)$ by its parabolic subgroup consisting of Lorentz transformations $\mathrm{SO}(3,1)(\mathrm{M})$, dilatations $\mathrm{SO}(1,1)(\mathrm{A})$ and conformal boosts (N). The resulting space is $\overline{\mathbb{M}}^{4}=\left(S^{1} \times S^{3}\right) / \mathbb{Z}^{2}$. The non-compact space is the coset $\mathrm{K} \backslash \mathrm{G}$ $=(\mathrm{SO}(4) \times \mathrm{SO}(2)) \backslash \mathrm{SO}(4,2) \sim \mathrm{S}(\mathrm{U}(2) \times \mathrm{U}(2)) \backslash \mathrm{SU}(2,2)$. It is known [16] that this space is a bounded complex domain whose boundary is precisely $\overline{\mathrm{M}}^{4}$. Therefore we believe that our one-dimensional considerations in this paper give a good idea of what happens in four dimensions as well.

\subsubsection{The compact space of $R$ symmetry}

The symmetry group we are discussing is not just the conformal group $\mathrm{SO}(1,2)$ but its tensor product with the R symmetry group $\mathrm{SO}(3)$. Consequently, our conformal fields are UIRs of both groups. While it is customary to realize the UIRs of the conformal group in terms of fields, i.e., functions on a space-time, in physics we usually exhibit the $\mathrm{R}$ symmetry by attaching indices to the fields. However, for the purpose of studying the pp-wave contraction it is necessary to treat both symmetry groups on the same footing. Thus, we are lead to extend space-time by an auxiliary internal space, in which the $\mathrm{R}$ symmetry group acts.f Once again, it can be introduced as a coset of the $\mathrm{R}$ symmetry group. In our simple case there is only one such coset: the two-sphere $S^{2} \sim \mathrm{SO}(2) \backslash \mathrm{SO}(3) \sim \mathrm{U}(1) \backslash \mathrm{SU}(2)$, which is the simplest example of a compact complex manifold. Its stereographic projection is the complex plane $\mathbb{C}^{1}$ with the infinite point added.

In the four-dimensional case one has to divide the R symmetry group $\mathrm{SO}(6) \sim \mathrm{SU}(4)$ by one of its maximal compact subgroups, $\mathrm{K}=\mathrm{SO}(4) \times \mathrm{SO}(2) \sim \mathrm{S}(\mathrm{U}(2) \times \mathrm{U}(2))$, which coincides with the maximal compact subgroup of the conformal group $\mathrm{SO}(4,2)$. The resulting coset space is again a compact complex manifold.

\subsection{The light cone as an example of a homogeneous space}

Here we recall Dirac's "light ray" realization of the coset space described in 2.2.1 (see e.g. [20]). It serves as a good introduction to the coset realizations we use in Section 3.3.

\footnotetext{
${ }^{4}$ This idea is commonly used in the literature on representation theory (see, e.g., 16, 17]). As a side remark, note that it is in the basis of the so-called "harmonic superspace" approach to extended supersymmetry, see 18, 19].
} 
The starting point is the "light cone" (the positive cone $\mathrm{C}_{+}$in $\mathbb{R}^{1,2}$ ). It is described by a light-like vector $\xi^{a}, a=0,1,2$ :

$$
\xi^{a} \xi_{a}=-\left(\xi^{0}\right)^{2}+\left(\xi^{1}\right)^{2}-\left(\xi^{2}\right)^{2}=0,
$$

where $\xi_{a}=\eta_{a b} \xi^{b}$. This condition is invariant under the linear action of the conformal group $\mathrm{SO}(1,2)$. Its generators are given by

$$
M_{a b}=\xi_{a} \partial / \partial \xi^{b}-\xi_{b} \partial / \partial \xi^{a}
$$

and satisfy the algebra (1).

The conformal algebra can be realized on fields $\Phi(\xi)$ on the light cone, homogeneous of degree $\ell, \Phi(\rho \xi)=\rho^{\ell} \Phi(\xi)$. This condition effectively eliminates one degree of freedom, so we are left with a single independent variable. In this way we introduce the one-dimensional Minkowski coordinate (time) $x \equiv x^{0}$ as a projective coordinate:

$$
x=\frac{\xi^{0}}{\xi^{1}+\xi^{2}} .
$$

We have chosen to divide by the combination $\xi^{1}+\xi^{2}$ because it transforms homogeneously under dilatations, as can easily be verified. Thus, the new coordinate $x$ has a definite dilatation weight.

The Minkowski fields $\varphi(x)$ are then introduced through the relation

$$
\Phi(\xi)=\left(\xi^{1}+\xi^{2}\right)^{-\Delta} \varphi(x),
$$

where $\Delta=-\ell>0$ is the conformal dimension of the field. In this form it is obvious that $\Phi(\xi)$ is homogeneous of degree $\ell$, since the coordinate $x$ is inert under the rescaling $\xi^{a} \rightarrow \rho \xi^{a}$.

The principal difference between the fields $\Phi(\xi)$ and $\varphi(x)$ is that the former is manifestly covariant under the conformal group $\mathrm{SO}(1,2)$, while the latter transforms non-linearly. To see this, it is instructive to rewrite the generators (9) in terms of the single variable $x$. With the help of (8)-(11) it is not hard to derive the familiar form of the generators of the conformal algebra (2):

$$
\begin{aligned}
& D=x \frac{d}{d x}+\Delta \\
& P=\frac{1}{\sqrt{2}} \frac{d}{d x} \\
& K=\frac{1}{\sqrt{2}}\left(x^{2} \frac{d}{d x}+2 \Delta x\right)
\end{aligned}
$$

It is well known that such fields realize irreducible representations of the conformal group of weight $\Delta$. Indeed, on $\varphi(x)$ the Casimir (5) takes the value $C_{2}=\Delta(\Delta-1)$.

The construction above provides a realization of the coset space $\mathbb{R}^{1} \backslash \mathrm{C}_{+}$. We start with functions defined on the bigger space $\mathrm{C}_{+}$and impose a homogeneity condition with respect to the "stability group" $\mathbb{R}^{1} \sim \mathrm{SO}(1,1)$. The degree of homogeneity corresponds to the weight, i.e., to the representation label. The resulting fields depend on the "carrier" 
coordinate (in eq. (11) it is $\xi^{1}+\xi^{2}$ ) for the stability group through a factor determined by the weight. In what follows we shall apply this coset construction to the homogeneous spaces $\mathrm{K} \backslash \mathrm{G}$, where $\mathrm{K}=\mathrm{U}(1)$ and $\mathrm{G}=\mathrm{SU}(2)$ or $\mathrm{SU}(1,1)$. Our fields will be defined on the whole group $\mathrm{G}$, but they will be homogeneous under the action of the subgroup $\mathrm{K}$. The manifest covariance under $\mathrm{K}$ will be achieved at the expense of introducing an extra coordinate for it. However, the dependence on this "weight carrier" coordinate will always be factorized.

\section{Free conformal fields}

In this section we describe the lowest-weight UIRs of the conformal and R symmetry group using a spinor basis. Next we calculate the matrix elements of the representations and show that a particular subset of them can be used as an orthonormal basis for expanding free conformal fields of a given weight. Then we introduce different coordinatizations by decomposing the group elements according to the patterns discussed in Section 2.2.

\subsection{Lowest-weight unitary representations of $\mathrm{SU}(2)$ and $\mathrm{SU}(1,1)$}

We start by recalling some basic facts about the representations with lowest weight, commonly used in physics, both in the familiar case of $\mathrm{SU}(2)$ as well as the more elaborate case of SU(1,1). The representations of these two groups (and, more generally, of their common complex form $\mathrm{SL}(2, \mathbb{C})$ ) are most conveniently realized [21, 22, 15] in a vector space spanned by complex monomials:

$$
|a, b\rangle=z_{1}^{a} z_{2}^{b}
$$

It is well known that the UIRs of the compact group $\mathrm{SU}(2)$ are finite-dimensional and are given by the homogeneous polynomials of degree $2 \ell$ formed by the monomials:

$$
\mathrm{SU}(2): \quad|\ell, m\rangle=N_{m} z_{1}^{m} z_{2}^{2 \ell-m}, \quad \ell \geq 0, \quad m=0,1, \ldots, 2 \ell .
$$

In order to determine the normalization coefficients $N_{m}$, let us first write down the generators (6) explicitly:

$$
L_{0}=\frac{1}{2}\left(z_{1} \partial / \partial z_{1}-z_{2} \partial / \partial z_{2}\right), \quad L_{+}=\frac{1}{\sqrt{2}} z_{1} \partial / \partial z_{2}, \quad L_{-}=\frac{1}{\sqrt{2}} z_{2} \partial / \partial z_{1} .
$$

Their action on the basis vectors is given by

$$
\begin{aligned}
& L_{0}|\ell, m\rangle=(m-\ell)|\ell, m\rangle \\
& L_{+}|\ell, m\rangle=\frac{2 \ell-m}{\sqrt{2}} \frac{N_{m}}{N_{m+1}}|\ell, m+1\rangle \\
& L_{-}|\ell, m\rangle=\frac{m}{\sqrt{2}} \frac{N_{m}}{N_{m-1}}|\ell, m-1\rangle
\end{aligned}
$$

Note that $|\ell, m\rangle$ are eigenvectors of the $\mathrm{U}(1)$ generator $L_{0}$ with eigenvalue ("charge") $m-\ell$.

\footnotetext{
${ }^{5}$ Our presentation follows Ref. [15].
} 
Assuming that the basis is orthonormal,

$$
\langle\ell, m \mid \ell, n\rangle=\delta_{m n},
$$

we can translate the unitarity condition (đ) into a condition on the normalization coefficients $N_{m}$ :

$$
\left|\frac{N_{m+1}}{N_{m}}\right|^{2}=\frac{2 \ell-m}{m+1} \geq 0 .
$$

It has the following solution

$$
N_{m}=\left(\frac{(2 \ell) !}{m !(2 \ell-m) !}\right)^{1 / 2},
$$

where we have set $N_{0}=1$. We remark that the range of the index $m$ shown in (14) is in fact a consequence of unitarity, as is clear from eq. (18).

The case of the non-compact group $\mathrm{SU}(1,1)$ is considerably more involved. This group is known to possess four series of infinite-dimensional UIRs [23, 14]: principal, supplementary and two discrete series $\mathcal{D}_{\ell}^{ \pm}$. All of them admit a realization on vectors of the type (6). However, only the discrete series are suitable for applications in CFT, since only they have a lowest weight vector (for $\mathcal{D}_{\ell}^{+}$) or a highest weight vector (for $\mathcal{D}_{\ell}^{-}$), and, correspondingly, their energy spectrum is bounded below or above [24]. Here we restrict ourselves to the discrete series $\mathcal{D}_{\ell}^{+}$.

The first modification, compared to the case $\mathrm{SU}(2)$, is in the expression for the $\mathrm{SU}(1,1)$ generators (3) (cf. (15)):

$$
M_{0}=\frac{1}{2}\left(z_{1} \partial / \partial z_{1}-z_{2} \partial / \partial z_{2}\right), \quad M_{+}=-\frac{i}{\sqrt{2}} z_{1} \partial / \partial z_{2}, \quad M_{-}=-\frac{i}{\sqrt{2}} z_{2} \partial / \partial z_{1} .
$$

We use the same basis (14), but now keeping $m$ and $\ell$ arbitrary (in general, complex). After making the corresponding changes in (16), we see that if neither $m$ nor $2 \ell-m$ are integers, there can be no lowest (or highest) weight vector. This case corresponds to the principal or supplementary series of representations. The vector with $m=0$ is the lowest weight vector of the invariant subspace spanned by $m=0,1,2, \ldots$ This case corresponds to the discrete series $\mathcal{D}_{\ell}^{+}$.

Further, we have to examine the condition for unitarity, which now reads

$$
\left|\frac{N_{m+1}}{N_{m}}\right|^{2}=\frac{m-2 \ell}{m+1} \geq 0 .
$$

In the case of the discrete series $\mathcal{D}_{\ell}^{+} m=0,1,2, \ldots$, therefore we have to take a strictly negative weight $\ell<0$ (we exclude the trivial representation $m=\ell=0$ ). In addition, for our illustrative purposes it will be sufficient to restrict ourselves to integer or half-integer 
values of $\ell$ only. $]$ Thus, the vector space of the representation $\mathcal{D}_{\ell}^{+}$becomes (cf. (14))

$$
\mathrm{SU}(1,1): \quad|\ell, m\rangle=N_{m} z_{1}^{m} z_{2}^{2 \ell-m}, \quad \ell<0, \quad m=0,1, \ldots, \infty
$$

where

$$
N_{m}=\left(\frac{(m-2 \ell-1) !}{m !(-2 \ell-1) !}\right)^{1 / 2}
$$

We note an important difference between the finite-dimensional UIRs of $\mathrm{SU}(2)$ and the infinite-dimensional lowest weight UIRs of $\mathrm{SU}(1,1)$. The eigenvalue of the $\mathrm{U}(1)$ generator $L_{0}$ or $M_{0}$ on the lowest weight vector ("vacuum") $|\ell, 0\rangle$, which is $-\ell$ in both cases, is negative for $\mathrm{SU}(2)$ and positive for $\mathrm{SU}(1,1)$. So, this eigenvalue, which can be related to the physical quantity "energy" [24], stays positive within the infinite-dimensional space of an $\mathrm{SU}(1,1)$ UIR of the class $\mathcal{D}_{\ell}^{+}$. It is customary to label the UIRs of both groups by a positive number, the "isospin" $J=\ell$ for $\mathrm{SU}(2)$ and the "conformal weight" $\Delta=-\ell$ for $\mathrm{SU}(1,1)$. It then easily follows that the quadratic Casimirs (7) of $\mathrm{SU}(2)$ and (5) of $\mathrm{SU}(1,1)$ take the values $C_{2}^{S U(2)}=J(J+1)$ and $C_{2}^{S U(1,1)}=\Delta(\Delta-1)$.

\subsection{Matrix elements of $\mathrm{SU}(2)$ and $\mathrm{SU}(1,1)$}

The matrix elements of $\mathrm{SU}(2)$ or $\mathrm{SU}(1,1)$ in the bases (14) or (22) can be obtained by considering the pair of complex numbers $z_{1}, z_{2}$ as the fundamental spinor representation! of the complexified group $\mathrm{SL}(2, \mathbb{C})$. For any $\mathrm{SL}(2, \mathbb{C})$ matrix

$$
g=\left(\begin{array}{cc}
\alpha & \beta \\
\gamma & \delta
\end{array}\right), \quad \alpha \delta-\beta \gamma=1
$$

we have

$$
T(g)\left(\begin{array}{c}
z_{1} \\
z_{2}
\end{array}\right)=\left(\begin{array}{c}
\alpha z_{1}+\beta z_{2} \\
\gamma z_{1}+\delta z_{2}
\end{array}\right)
$$

Let us apply this rule to the basis vectors $|\ell, m\rangle$ and expand the result in the same basis:

$$
T^{\ell}(g)|\ell, m\rangle=N_{m}\left(\alpha z_{1}+\beta z_{2}\right)^{m}\left(\gamma z_{1}+\delta z_{2}\right)^{2 \ell-m}=\sum_{n} t_{m n}^{\ell}(g) N_{n} z_{1}^{n} z_{2}^{2 \ell-n} .
$$

Here the sum over $n$ runs from 0 to either $2 \ell$ (for $\mathrm{SU}(2))$ or $\infty$ (for $\mathrm{SU}(1,1)$ ). The matrices $t_{m n}^{\ell}(g)$ are the matrix elements of the representation of weight $\ell$ corresponding to the group element $g(24)$. Later on we shall specify $g$ to an $\mathrm{SU}(2)$ or an $\mathrm{SU}(1,1)$ matrix written down in different parametrizations, but for the moment we keep the $\operatorname{SL}(2, \mathbb{C})$ notation.

\footnotetext{
${ }^{6}$ We remark that the name "discrete series" comes from precisely this restriction, which is common in the mathematical literature [23, 14. Allowing for non-integer weight $2 \ell$ (or "anomalous dimension" in the physics terminology) leads to mathematical subtleties which we need not discuss here. If one insists on the representations of the algebra being integrable to representations of the group, one is confronted with infinitely multivalued functions 25]. Another way to see the problem is to switch from the group SU(1,1) to its infinitely sheeted universal covering [24, 26]. For a discussion from the physicist's point of view see also Ref. 27.

${ }^{7}$ The group $\mathrm{SL}(2, \mathbb{C})$ has a second fundamental representation obtained by complex conjugation.
} 
Now, consider the subgroup $\mathrm{U}(1) \times \mathrm{SO}(1,1) \subset \mathrm{SL}(2, \mathbb{C})$, i.e., the group of diagonal matrices

$$
h=\left(\begin{array}{ll}
\alpha^{1 / 2} & 0 \\
0 & \alpha^{-1 / 2}
\end{array}\right)
$$

From (26) we have

$$
t_{m n}^{\ell}(h)=\delta_{m n} \alpha^{m-\ell} .
$$

Thus, using the group multiplication property, we obtain the transformation law of the matrix elements $t_{m n}^{\ell}(g)$ under the left action of the subgroup (27):

$$
t_{m n}^{\ell}(h g)=\sum_{k} t_{m k}^{\ell}(h) t_{k n}^{\ell}(g)=\alpha^{m-\ell} t_{m n}^{\ell}(g) .
$$

In particular, setting $m=0$, we find

$$
t_{0 n}^{\ell}(h g)=\alpha^{-\ell} t_{0 n}^{\ell}(g) .
$$

The conclusion is that the matrix elements $t_{0 n}^{\ell}(g)$, which correspond to the lowest weight vector, transform homogeneously with weight $-\ell$.

Another important property of the matrix elements $t_{m n}^{\ell}(g)$ is their orthogonality with respect to a suitable scalar product in the group space. Moreover, under certain conditions they form complete bases for expanding whole classes of functions on the group or on its factor spaces. A well-known example is provided by the spherical harmonic functions for $\mathrm{SU}(2)$. In our notation they correspond to the matrix elements

$$
Y_{\ell, \ell-n} \leftrightarrow t_{\ell n}^{\ell}
$$

They are used for expanding (square integrable) functions on the sphere $S^{2}$, invariant under the action of $\mathrm{SU}(2)$ ("scalar fields"). Indeed, from (29) it is clear that $t_{\ell n}^{\ell}$ have vanishing weight. Note that an expansion in terms of the spherical harmonics constitutes an infinitely reducible representation of $\mathrm{SU}(2)$. What we need here is different.

As explained at the end of Section 2.3, for us the conformal fields are defined as functions on the group $\mathrm{G}$ transforming homogeneously under the left action of the subgroup $\mathrm{K}$ and being irreducible under both $\mathrm{G}$ and $\mathrm{K}$. This gives an effective realization of the coset $\mathrm{K} \backslash \mathrm{G}$. In this paper we consider the cosets $\mathrm{U}(1) \backslash \mathrm{SU}(2)$ and $\mathrm{U}(1) \backslash \mathrm{SU}(1,1)$. In both cases $\mathrm{K}$ is diagonal, so the discussion above applies. So, we deduce that such functions can be expanded in terms of the matrix elements $t_{0 n}^{\ell}(g)$ corresponding to the lowest weight vector of the representation $T^{\ell}(g)$ :

$$
\Phi^{\ell}(g)=\sum_{n} a_{n} t_{0 n}^{\ell}(g)
$$

Under the diagonal subgroup $h(27)$ they transform homogeneously with weight $-\ell$ :

$$
\Phi^{\ell}(h g)=\alpha^{-\ell} \Phi^{\ell}(g) .
$$

At the same time, the positive number $|\ell|$ labels the UIR of the group $g$ realized on the function $\Phi^{\ell}$. 
The matrix elements $t_{0 n}^{\ell}(g)$ are particularly easy to derive. Indeed, setting $m=0$ in (26) we obtain

$$
\left(\gamma z_{1}+\delta z_{2}\right)^{2 \ell}=\sum_{n} t_{0 n}^{\ell}(g) N_{n} z_{1}^{n} z_{2}^{2 \ell-n}
$$

The expansion of the left-hand side is performed differently for $\mathrm{SU}(2)$ and $\mathrm{SU}(1,1)$. In the case of $\mathrm{SU}(2)$ we have $\ell \geq 0$, so this is just a binomial expansion, valid for any values of $\gamma, \delta$ and $z_{1,2}$. After equating the coefficients of the terms $z_{1}^{n} z_{2}^{2 \ell-n}$, we find

$$
\mathrm{SU}(2): \quad t_{0 n}^{\ell}(g)=\left(\frac{(2 \ell) !}{n !(2 \ell-n) !}\right)^{1 / 2} \gamma^{n} \delta^{2 \ell-n} .
$$

In the case of $\mathrm{SU}(1,1)$ we have $\ell<0$, so we need to define the parameter domain in which we can expand. For instance, we can set

$$
|\gamma|<|\delta|, \quad\left|z_{1}\right|<\left|z_{2}\right|
$$

and rewrite the left-hand side of eq. (34) as follows:

$$
\left(\gamma z_{1}+\delta z_{2}\right)^{2 \ell}=\left(\delta z_{2}\right)^{2 \ell}\left(1+\frac{\gamma}{\delta} \frac{z_{1}}{z_{2}}\right)^{-2|\ell|}
$$

and then expand. As a result, we obtain the matrix elements

$$
\mathrm{SU}(1,1): \quad t_{0 n}^{\ell}(g)=(-1)^{n}\left(\frac{(n-2 \ell-1) !}{n !(-2 \ell-1) !}\right)^{1 / 2} \gamma^{n} \delta^{2 \ell-n}
$$

\subsection{Coordinates on the homogeneous spaces $\mathrm{U}(1) \backslash \mathrm{SU}(2)$ and $\mathrm{U}(1) \backslash \mathrm{SU}(1,1)$}

As explained above, our conformal fields are defined on coset spaces of the conformal group $\mathrm{SU}(1,1)$ and the $R$ symmetry group SU(2). The matrix elements introduced in the preceding subsection provide a natural basis for expanding such functions.

\subsubsection{The compact case}

Consider first the left coset $\mathrm{U}(1) \backslash \mathrm{SU}(2) \sim S^{2}$. We can introduce coordinates on it by specifying the matrix (24) to $\mathrm{SU}(2)$ and writing it down in a factorized form, where the $\mathrm{U}(1)$ factor stands on the left:

$$
g=\left(\begin{array}{cc}
e^{i \phi / 2} & 0 \\
0 & e^{-i \phi / 2}
\end{array}\right) \frac{1}{\sqrt{1+|\tau|^{2}}}\left(\begin{array}{rr}
1 & -\bar{\tau} \\
\tau & 1
\end{array}\right) .
$$

Here $-2 \pi \leq \phi<2 \pi$ is the coordinate of the subgroup $\mathrm{U}(1)$ and $\tau$ is the complex stereographic coordinate on the coset $\mathrm{U}(1) \backslash \mathrm{SU}(2) \sim S^{2}$. Reading $\gamma$ and $\delta$ off from (39) and substituting in (35), we obtain

$$
t_{0 n}^{\ell}=\left(\frac{(2 \ell) !}{n !(2 \ell-n) !}\right)^{1 / 2} e^{-i \ell \phi} \frac{\tau^{n}}{\left(1+|\tau|^{2}\right)^{\ell}} .
$$


According to our definition (32), a "field", i.e. a function carrying the irreducible representation of lowest weight $-\ell$, has the form

$$
\Phi^{\ell}(\tau, \bar{\tau}, \phi)=\sum_{n=0}^{2 \ell} a_{n} t_{0 n}^{\ell}(\tau, \bar{\tau}, \phi)=\frac{e^{-i \ell \phi}}{\left(1+|\tau|^{2}\right)^{\ell}} \sum_{n=0}^{2 \ell} b_{n} \tau^{n} \equiv e^{-i \ell \phi} \varphi(\tau, \bar{\tau}) .
$$

We clearly see that the dependence on the $\mathrm{U}(1)$ coordinate $\phi$ is given by an overall weight factor, which reflects the fact that the field $\Phi^{\ell}$ is effectively defined on the coset $\mathrm{U}(1) \backslash \mathrm{SU}(2) \sim$ $S^{2}$. In a way, the picture is very similar to Dirac's light-cone description of conformal fields: Eq. (41) is the analog of eq. (11). The important difference, however, is that the factor in the right-hand side of eq. (41) comes from the compact subgroup $\mathrm{SO}(2)$, while that in eq. (11) is related to the non-compact $\mathrm{SO}(1,1)$.

Further, the dependence on the complex coordinates $\tau, \bar{\tau}$ in (41) is essentially holomorphic, i.e. it is determined by a polynomial $\sum_{n=0}^{2 \ell} b_{n} \tau^{n}$ of degree $2 \ell$. The non-holomorphic factor $1 /\left(1+|\tau|^{2}\right)^{\ell}$ can be combined with the SU(2) invariant measure. The scalar product in the space of the functions (41) is given by

$$
\begin{aligned}
\left\langle\Phi_{1}^{\ell} \Phi_{2}^{\ell^{\prime}}\right\rangle & =\frac{\sqrt{(2 \ell+1)\left(2 \ell^{\prime}+1\right)}}{4 \pi^{2}} \int_{\mathbb{C}} \frac{d \tau d \bar{\tau}}{\left(1+|\tau|^{2}\right)^{2}} \int_{-2 \pi}^{2 \pi} d \phi \Phi_{1}^{\ell} \bar{\Phi}_{2}^{\ell^{\prime}} \\
& =\frac{2 \ell+1}{\pi} \delta^{\ell \ell^{\prime}} \int_{\mathbb{C}} \frac{d \tau d \bar{\tau}}{\left(1+|\tau|^{2}\right)^{2}} \varphi_{1}(\tau, \bar{\tau}) \bar{\varphi}_{2}(\tau, \bar{\tau}) .
\end{aligned}
$$

It is easy to check that the matrix elements (40) form an orthonormal set with respect to this scalar product:

$$
\left\langle t_{0 n}^{\ell} t_{0 n^{\prime}}^{\ell^{\prime}}\right\rangle=\delta^{\ell \ell^{\prime}} \delta_{n n^{\prime}}
$$

Let us address the question of how the $\mathrm{SU}(2)$ algebra is realized on such functions. By construction, they are invariant under the right action of G. To put it differently, the right action on the coordinates can be compensated by a suitable transformation of the coefficients $b_{n}$ in (41), which form a UIR of G on their own. Then it is natural to realize the $\mathrm{SU}(2)$ algebra in terms of the left-covariant Lie derivatives on the coset $\mathrm{K} \backslash \mathrm{G}$. Their explicit form can be derived using the standard Cartan method of differential forms. It consists in expanding the form $g^{-1} d g$ in the basis of the Lie algebra generators

$$
L_{0}=-\frac{1}{2}\left(\begin{array}{rr}
1 & 0 \\
0 & -1
\end{array}\right), \quad L_{+}=\frac{1}{\sqrt{2}}\left(\begin{array}{ll}
0 & 0 \\
1 & 0
\end{array}\right), \quad L_{-}=\frac{1}{\sqrt{2}}\left(\begin{array}{ll}
0 & 1 \\
0 & 0
\end{array}\right)
$$

and then inverting the coefficient matrix. An alternative way (the simplest) is to directly compute the left action of the group on the matrices (39) and extract from it the action of the generators (44) on the coordinates. Yet another way [18], which is suitable for making contact with the discussion in Section 2.3, is to realize the generators in terms of the entries $g_{ \pm}{ }^{i}$ of an abstract $\mathrm{SU}(2)$ matrix. Here \pm refers to the first or second row of the matrix, according to the sign in $e^{ \pm i \psi / 2}$. They satisfy the unit determinant condition

$$
g_{+}{ }^{i} \epsilon_{i j} g_{-}^{j}=1
$$

\footnotetext{
${ }^{8} \mathrm{An}$ analogy is provided by a four-dimensional scalar field written down as a power expansion, $\phi\left(x^{\mu}\right)=$ $f+f_{\mu} x^{\mu}+f_{\mu \nu} x^{\mu} x^{\nu}+\ldots$ The coefficients in this expansion $f, f_{\mu}, f_{\mu \nu}, \ldots$ are a Lorentz scalar, vector, tensor, etc., although the field $\phi\left(x^{\mu}\right)$ itself is a Lorentz scalar.
} 
Now, let us write down the following differential operators:

$$
\begin{aligned}
& L_{0}=g_{+}{ }^{i} \frac{\partial}{\partial g_{+}{ }^{i}}-g_{-}{ }^{i} \frac{\partial}{\partial g_{-}{ }^{i}}, \\
& L_{-}=g_{-}{ }^{i} \frac{\partial}{\partial g_{+}{ }^{i}}, \quad L_{+}=g_{+}{ }^{i} \frac{\partial}{\partial g_{-}{ }^{i}} .
\end{aligned}
$$

Here we can formally treat the variables $g_{ \pm}{ }^{i}$ as independent, since all the three operators are compatible with the condition (45). Then it is almost obvious that they form the $\mathrm{SU}(2)$ algebra (6). By construction, these generators are right-invariant (the right-hand side indices $i$ of $g_{ \pm}{ }^{i}$ are contracted), but they have charges under the left group. It is then a straightforward exercise to substitute the explicit coordinatization (39) and obtain the left covariant derivatives:

$$
\begin{aligned}
& L_{0}=-i \frac{\partial}{\partial \phi} \\
& L_{-}=\frac{e^{-i \phi}}{\sqrt{2}}\left[i\left(1+|\tau|^{2}\right) \frac{\partial}{\partial \bar{\tau}}-\tau \frac{\partial}{\partial \phi}\right] \\
& L_{+}=\frac{e^{i \phi}}{\sqrt{2}}\left[i\left(1+|\tau|^{2}\right) \frac{\partial}{\partial \tau}+\bar{\tau} \frac{\partial}{\partial \phi}\right] .
\end{aligned}
$$

Applying the first two of these generators to the field $\Phi^{\ell}(41)$, we see that it can be regarded as a lowest weight vector of the left algebra (47), in accord with our definition of the field:

$$
L_{0} \Phi^{\ell}=-\ell \Phi^{\ell}, \quad L_{-} \Phi^{\ell}=0 .
$$

We stress once more that our fields $\Phi^{\ell}(\tau, \bar{\tau}, \phi)$ (41) are defined as homogeneous functions on the whole of $\mathrm{SU}(2)$. If we want to deal with functions on the sphere $S^{2} \sim \mathrm{U}(1) \backslash \mathrm{SU}(2)$ itself, we need a different realization of the $\mathrm{SU}(2)$ algebra, this time corresponding to the right action of the group. The situation here is similar to Section 2.3. There we started with a manifestly covariant form of the $\mathrm{SO}(1,2)$ generators, eq. (9). In fact, the vector $\xi^{a}$ can be regarded as a combination of two rows of an $\mathrm{SO}(1,2)$ matrix $g_{A}{ }^{a}$ having definite $\mathrm{SO}(1,1)$ weight under the left action of the group, $\xi^{a}=g_{0}{ }^{a}+g_{2}{ }^{a}$ (the light-cone condition (8) then follows from the pseudo-orthogonality of this matrix). The operators $M_{a b}$ are constructed so that they have vanishing left weight, but are manifestly covariant under the right action. By analogy, in the case of $\mathrm{SU}(2)$ we introduce the generators

$$
L^{i}{ }_{j}=g_{+}{ }^{i} \frac{\partial}{\partial g_{+}{ }^{j}}-g_{-}{ }^{i} \frac{\partial}{\partial g_{-}{ }^{j}}-\text { trace in } i, j .
$$

Once again, they form the algebra of $\mathrm{SU}(2)$, but this time written in spinor notation. Like (9), these generators are left-invariant, but right-covariant. Substituting the explicit coordinatization (39), changing from spinor to vector notation and applying the generators to the function $\varphi(\tau, \bar{\tau})$ in (41) (cf. (11)), we obtain the right algebra realized on functions 
on the sphere $S^{2}$ :

$$
\begin{aligned}
& \widetilde{L}_{0}=\tau \frac{\partial}{\partial \tau}-\bar{\tau} \frac{\partial}{\partial \bar{\tau}}-\ell \\
& \widetilde{L}_{+}=\frac{i}{\sqrt{2}}\left[\frac{\partial}{\partial \bar{\tau}}+\tau^{2} \frac{\partial}{\partial \tau}-\ell \tau\right] \\
& \widetilde{L}_{-}=\frac{i}{\sqrt{2}}\left[\frac{\partial}{\partial \tau}+\bar{\tau}^{2} \frac{\partial}{\partial \bar{\tau}}+\ell \bar{\tau}\right]
\end{aligned}
$$

\subsubsection{The non-compact case}

The case of $\mathrm{SU}(1,1)$ can be treated in the same way. We consider the left coset $\mathrm{U}(1) \backslash \mathrm{SU}(1,1) \sim$ $\mathcal{D}^{1} \sim \mathbb{H}_{+}$(see Section 2.2.2). Now the factorization (39) is replaced by

$$
g=\left(\begin{array}{cc}
e^{i \psi / 2} & 0 \\
0 & e^{-i \psi / 2}
\end{array}\right) \frac{1}{\sqrt{1-|t|^{2}}}\left(\begin{array}{cc}
1 & \bar{t} \\
t & 1
\end{array}\right), \quad|t|<1,
$$

where the complex variable $t$ describes the interior of the unit disc. The analogs of eqs. (40), (41) and (42) are

$$
\begin{gathered}
t_{0 n}^{\ell}=(-1)^{n}\left(\frac{(n-2 \ell-1) !}{n !(-2 \ell-1) !}\right)^{1 / 2} e^{-i \ell \psi} \frac{t^{n}}{\left(1-|t|^{2}\right)^{\ell}} \\
\Phi^{\ell}(t, \bar{t}, \psi)=\sum_{n=0}^{\infty} a_{n} t_{0 n}^{\ell}(t, \bar{t}, \psi)=\frac{e^{-i \ell \psi}}{\left(1-|t|^{2}\right)^{\ell}} \sum_{n=0}^{\infty} b_{n} t^{n} \equiv e^{-i \ell \psi} \varphi(t, \bar{t})
\end{gathered}
$$

and

$$
\left\langle\Phi_{1}^{\ell} \Phi_{2}^{\ell^{\prime}}\right\rangle=\frac{\sqrt{(2 \ell+1)\left(2 \ell^{\prime}+1\right)}}{4 \pi^{2}} \int_{|t|<1} \frac{d t d \bar{t}}{\left(1-|t|^{2}\right)^{2}} \int_{-2 \pi}^{2 \pi} d \psi \Phi_{1}^{\ell} \bar{\Phi}_{2}^{\ell^{\prime}},
$$

where the positive value of the square root is assumed in the r.h.s. This time the field is determined by the function $\sum_{n=0}^{\infty} b_{n} t^{n}$, holomorphic inside the unit disc, and the factor $1 /\left(1-|t|^{2}\right)^{\ell}$ can be combined with the $\mathrm{SU}(1,1)$ invariant measure. The left-covariant derivatives on the coset $\mathrm{U}(1) \backslash \mathrm{SU}(1,1)$ are given by (cf. (47))

$$
\begin{aligned}
& M_{0}=-i \frac{\partial}{\partial \psi} \\
& M_{-}=\frac{e^{-i \psi}}{\sqrt{2}}\left[i\left(1-|t|^{2}\right) \frac{\partial}{\partial \bar{t}}+t \frac{\partial}{\partial \psi}\right] \\
& M_{+}=\frac{e^{i \psi}}{\sqrt{2}}\left[i\left(1-|t|^{2}\right) \frac{\partial}{\partial t}-\bar{t} \frac{\partial}{\partial \psi}\right] .
\end{aligned}
$$

As before (cf. (48)), the fields $\Phi^{\ell}$ are lowest weight vectors for the left algebra (55):

$$
M_{0} \Phi^{\ell}=-\ell \Phi^{\ell}, \quad M_{-} \Phi^{\ell}=0 .
$$


We can switch from homogeneous functions on the whole of $\mathrm{SU}(1,1)$ to functions $\varphi(t, \bar{t})$ (53) on the disc $\mathcal{D}^{1}$ by considering the right action of $\mathrm{SU}(1,1)$. The generators of the right algebra realized on functions on the disc are

$$
\begin{aligned}
& \widetilde{M}_{0}=t \frac{\partial}{\partial t}-\bar{t} \frac{\partial}{\partial \bar{t}}-\ell \\
& \widetilde{M}_{+}=\frac{i}{\sqrt{2}}\left[\frac{\partial}{\partial \bar{t}}-t^{2} \frac{\partial}{\partial t}+\ell t\right] \\
& \widetilde{M}_{-}=\frac{i}{\sqrt{2}}\left[\frac{\partial}{\partial t}-\vec{t}^{2} \frac{\partial}{\partial \bar{t}}-\ell \bar{t}\right] .
\end{aligned}
$$

\subsection{Minkowski space as a coset space}

The realization of the conformal group in terms of holomorphic functions in the complex coset space $\mathrm{U}(1) \backslash \mathrm{SU}(1,1)$ is very convenient for studying the UIRs. However, in physics we are accustomed to a different realization, in real Minkowski space. A characteristic feature of this description is that the fields $\phi(x)$ transform homogeneously under the non-compact dilatation subgroup $\mathrm{SO}(1,1) \subset \mathrm{SU}(1,1)$, and not under the compact $\mathrm{SO}(2) \subset \mathrm{SU}(1,1)$, as was the case in the preceding subsection. There are many papers and books where this subject is treated in great detail (see, e.g., [20, 17, 26]). Here we want to present a somewhat different picture, along the lines of Section 3.3. Our aim is to find a set of basis vectors for Minkowski fields, manifestly covariant under $\mathrm{SO}(1,1)$. This will be used in the discussion of the contraction mechanism in Section 6 . Our presentation is rather heuristic, a rigorous treatment can be found in 14.

The group $\mathrm{SU}(1,1)$ admits several decompositions. The Cartan decomposition KAK, where $\mathrm{K}=\mathrm{SO}(2)$ and $\mathrm{A}=\mathrm{SO}(1,1)$ is the factorization (51) of the $\mathrm{SU}(1,1)$ matrix. The Iwasawa decomposition $\mathrm{G}=\mathrm{KAN}$ is

$$
g=\left(\begin{array}{cc}
e^{i \theta / 2} & 0 \\
0 & e^{-i \theta / 2}
\end{array}\right)\left(\begin{array}{cc}
\cosh \frac{a}{2} & i \sinh \frac{a}{2} \\
-i \sinh \frac{a}{2} & \cosh \frac{a}{2}
\end{array}\right)\left(\begin{array}{cc}
1+i \frac{b}{2} & \frac{b}{2} \\
-\frac{b}{2} & 1-i \frac{b}{2}
\end{array}\right),
$$

where $a, b$ are real variables and $-2 \pi \leq \theta<2 \pi$. Here the first matrix is the $\mathrm{SO}(2)$ subgroup, the second is the Abelian factor A (dilatations, or the subgroup $\mathrm{SO}(1,1)$ ) and the third is the nilpotent factor $\mathrm{N}$ (corresponding to conformal boosts). This decomposition allows us to define the compactified Minkowski space as the boundary $S^{1}$ of the non-compact coset $\mathcal{D}^{1}=\mathrm{K} \backslash \mathrm{G}$. The group $\mathrm{K}$ acts transitively on $S^{1}$ : The stability subgroup of $\mathrm{K}$ at unity consists of two elements $I$ and $-I$, where $I$ is the $2 \times 2$ identity matrix. Thus, $S^{1} \sim$ $\mathrm{K} /\{I,-I\}$. The familiar Minkowski space coordinate $x \in \mathbb{R}^{1}$ can be introduced analytically by using the Cayley transform:

$$
e^{i \theta}=\frac{x-i}{x+i}
$$

This construction works equally well in the case $\mathrm{G}=\mathrm{SU}(2,2)$ and leads to the definition

of the compactified Minkowski space $\overline{\mathrm{M}}^{4}$ as a homogeneous space of the maximal compact subgroup $\mathrm{K}=\mathrm{SU}(2) \times \mathrm{SU}(2) \times \mathrm{U}(1)$.

Since we want to define a real space, it is preferable to work with the group of real matrices $\mathrm{SL}(2, \mathbb{R}) \sim \mathrm{SU}(1,1)$. In our specific one-dimensional situation there is another possibility 
to introduce the compactified Minkowski space. It is based on the Iwasawa decomposition $\mathrm{G}=\mathrm{NAK}$ of $\mathrm{SL}(2, \mathbb{R})$ :

$$
g=\left(\begin{array}{ll}
1 & 0 \\
b & 1
\end{array}\right)\left(\begin{array}{cc}
e^{a / 2} & 0 \\
0 & e^{-a / 2}
\end{array}\right)\left(\begin{array}{rr}
\cos \theta & \sin \theta \\
-\sin \theta & \cos \theta
\end{array}\right),
$$

where $a, b$ are real variables and $-\pi \leq \theta \leq \pi$. Here the first matrix is the nilpotent factor $N$ (corresponding to conformal boosts), the second is the Abelian factor $A$ (dilatations, or the subgroup $\mathrm{SO}(1,1) \subset \mathrm{SL}(2, \mathbb{R})$ ) and the third is the compact factor $\mathrm{K}$ (the subgroup $\mathrm{SO}(2) \subset \mathrm{SL}(2, \mathbb{R}))$. This decomposition allows us to define the compactified Minkowski space as the coset NA $\backslash \mathrm{G}$ with the additional requirements that the nilpotent factor $\mathrm{N}$ (the conformal boosts) acts trivially on the fields. Then it is clear from eq. (60) that the coset representative is just the third factor, i.e., the group $\mathrm{SO}(2)$ or the unit circle $S^{1}$. This time the Minkowski space coordinate $x \in \mathbb{R}^{1}$ can be introduced analytically by changing from the angular variable $\theta$ on the semicircle $-\pi / 2<\theta<\pi / 2$ to the real variable $x=\tan \theta$, $-\infty<x<\infty$. P We stress again that identifying $\mathrm{K}$ with the compactified Minkowski space is specific to $\mathrm{SL}(2, \mathbb{R})$.

Alternatively, we can introduce Minkowski space through the Bruhat decomposition NAMÑ (see, e.g., [17):

$$
g=\left(\begin{array}{ll}
1 & b \\
0 & 1
\end{array}\right)\left(\begin{array}{cc}
e^{a / 2} & 0 \\
0 & e^{-a / 2}
\end{array}\right)(-1)^{\epsilon}\left(\begin{array}{ll}
1 & 0 \\
x & 1
\end{array}\right),
$$

where $\epsilon=0,1$, for almost all matrices from $\operatorname{SL}(2, \mathbb{R})$. The factor $M=\{\mathbb{I},-\mathbb{I}\}$ in eq. (61) is the center of the group. Then Minkowski space can be identified with the coset $\mathrm{NAM} \backslash \mathrm{G}$. Thus, the familiar coordinate $x$ parametrizes the right nilpotent factor in (61), which generates translations. The left nilpotent factor in (61) generates conformal boosts and is part of the so-called "stability subgroup" of the origin $x=0$. The rest of it is the Abelian factor A (dilatations) and the factor $\mathrm{M}$ (if the space-time dimension is greater than one, $\mathrm{M}$ becomes the Lorentz group $\mathrm{SO}(d-1,1))$.

We recall the point of view on the conformal fields that we have adopted in this article. We treat them as functions on the conformal group $\mathrm{G}$ homogeneous under the left action of some subgroup $H$. Thus, they effectively live on the coset space $H \backslash G$. In the case at hand we have $\mathrm{H}=\mathrm{NAM}$. In CFT one imposes the additional requirement that the boosts $\mathrm{N}$ are represented trivially on the physical fields, $T_{N} \Phi(0)=\Phi(0)$. This amounts to setting $b=0$ in (61). Then, ignoring the constant center element $\mathrm{M}$, we see that our conformal fields can be viewed as functions of the lower triangular matrices

$$
g_{0}=\left(\begin{array}{cc}
e^{a / 2} & 0 \\
e^{-a / 2} x & e^{-a / 2}
\end{array}\right)
$$

homogeneous under the dilatations with parameter $a$. We remark that this realization of Minkowski space is closely related to Dirac's projective cone from Section 2.3. Indeed, the variable $a$ in (62) corresponds to the weight factor $\xi^{1}+\xi^{2}$, see (10) and (11).

The next step is to find a suitable orthogonal basis for conformal fields with manifest action of the dilatation group $\mathrm{SO}(1,1)$. This can be done by adapting the discussion of

\footnotetext{
${ }^{9}$ The compact coset space $\mathrm{NA} \backslash \mathrm{G}$ can be restored by adding the infinite point to the real line $\mathbb{R}^{1}$.
} 
Section 3.3 with some important modifications. The details are given in Appendix A. Here we only present the result. Since $\mathrm{SO}(1,1)$ is a non-compact group, the basis is labelled by a continuous complex variable $\nu$. The basis vectors are

$$
t_{+}^{\ell}(0, \nu ; a, x)=C(\nu) e^{-a \ell} x_{+}^{\nu}, \quad 2 \ell<\Re(\nu)<0,
$$

restricted to positive values of $x$ only, and similar vectors $t_{-}^{\ell}$ for $x<0$. Then a field of dilatation weight $\ell$ is given by the integral expansion (Mellin transform)

$$
\Phi^{\ell}(a, x)=\frac{1}{2 \pi i} \int_{c-i \infty}^{c+i \infty} d \nu\left[b_{+}(\nu) t_{+}^{\ell}(0, \nu ; a, x)+b_{-}(\nu) t_{-}^{\ell}(0, \nu ; a, x)\right] \equiv e^{-\ell a} \varphi(x) .
$$

The last term in this equation is the analog of eq. (11) from Dirac's description of the conformal fields.

\section{Contraction of conformal fields}

In this section we discuss the main point of the paper: How to perform the simultaneous contraction of the algebras of the conformal group $\mathrm{SU}(1,1)$ and the $\mathrm{R}$ symmetry group $\mathrm{SU}(2)$ in terms of conformal fields. We show that, starting from the very simple geometric idea of expanding the unit disc, one can derive, step by step, all the ingredients of the abstract algebraic contraction underlying the pp-wave limit.

\subsection{Expansion of the unit disc}

As explained in Section 3.3, the free conformal fields can be defined on the unit disc $\mathcal{D}^{1}$ parametrized by a complex coordinate $t,|t|<1$. The geometric idea behind the pp-wave limit is very simple: We want to rescale $t$, so that the radius of the disc becomes infinite and the disc covers the whole complex plane $\mathbb{C}^{1}$. To this end we introduce the contraction parameter $\Omega>0$ :

$$
t \rightarrow \Omega t \Rightarrow|t|<\frac{1}{\Omega}
$$

Letting $\Omega \rightarrow 0$, we achieve the desired result. So, we define the pp-wave limit as the "expansion" of the space in which the conformal algebra is realized.

Let us now see how this affects the conformal fields in the form (53). The weight $\ell$ in the non-compact case is negative, but in physics we are used to positive weights, therefore we replace $-\ell=\Delta>0$. Then we rewrite the basis vectors (52) in the form

$$
t_{0 n}^{\Delta} \equiv e^{i \Delta \psi} f_{n}^{\Delta}(t, \bar{t})=e^{i \Delta \psi} \frac{(-1)^{n}}{\sqrt{n !}}\left(\frac{\Gamma(2 \Delta+n)}{\Gamma(2 \Delta)}\right)^{1 / 2}\left(1-|t|^{2}\right)^{\Delta} t^{n} .
$$

We will take care of the phase factor $e^{i \Delta \psi}$ a bit later, for the moment we concentrate on $f_{n}^{\Delta}(t, \bar{t})$. Under the rescaling (65) $t^{n}$ goes to zero like $\Omega^{n}$, so we ought to compensate this by an appropriate behavior of the normalization factor in (66). Setting

$$
\Delta=\frac{\lambda}{2 \Omega^{2}},
$$


where $\lambda>0$ is a fixed constant, and using the asymptotic expressions

$$
\left(\frac{\Gamma\left(\lambda / \Omega^{2}+n\right)}{\Gamma\left(\lambda / \Omega^{2}\right)}\right)^{1 / 2} \approx \frac{\lambda^{n / 2}}{\Omega^{n}}, \quad\left(1-\Omega^{2}|t|^{2}\right)^{\lambda / 2 \Omega^{2}} \approx e^{-\frac{1}{2} \lambda|t|^{2}}
$$

valid for $\Omega \rightarrow 0$, we easily find

$$
\mathbf{f}_{n}=\lim _{\Omega \rightarrow 0} f_{n}^{\Delta}=(-1)^{n} \sqrt{\frac{\lambda^{n}}{n !}} t^{n} e^{-\frac{1}{2} \lambda|t|^{2}} .
$$

Note that the constant $\lambda$ can be absorbed into the complex variable by the rescaling $t \rightarrow$ $\sqrt{\lambda} t$, so

$$
\mathbf{f}_{n}=\frac{(-1)^{n}}{\sqrt{n !}} t^{n} e^{-\frac{1}{2}|t|^{2}}
$$

We conclude that what we have obtained in this well-defined contraction limit is the familiar Fock-Bargmann basis for the Heisenberg algebra.

The next question is what to do with the phase factor $e^{i \Delta \psi}$ in (66), which diverges when $\Delta \sim 1 / \Omega^{2}$. At this point we should remember that our conformal fields are defined as UIRs of both the conformal and R symmetry groups. In other words, they can be regarded as functions on $\mathrm{SU}(1,1) \times \mathrm{SU}(2)$, homogeneous under the left action of the subgroup $\mathrm{U}(1) \times \mathrm{U}(1)$ or, equivalently, as functions on $\mathcal{D}^{1} \times S^{2}$. Combining eqs. (41) and (53) and denoting the $\mathrm{SU}(2)$ weight $\ell=J>0$ ("isospin"), we can write down

$$
\Phi^{\Delta, J}(t, \bar{t}, \psi ; \tau, \bar{\tau}, \phi)=\sum_{n=0}^{\infty} \sum_{m=0}^{2 J} a_{m n} t_{0 n}^{\Delta}(t, \bar{t}, \psi) t_{0 m}^{J}(\tau, \bar{\tau}, \phi)=e^{i(\Delta \psi-J \phi)} \varphi(t, \bar{t}, \tau, \bar{\tau}) .
$$

Now we see two distinct possibilities to render the phase factor convergent. The first consists in rescaling each of two $\mathrm{U}(1)$ coordinates as follows:

$$
\psi=\Omega^{2} \tilde{\psi}, \quad \phi=\Omega^{2} \tilde{\phi}, \quad \lim _{\Omega \rightarrow 0} e^{i \Delta \psi}=e^{i \lambda \tilde{\psi}}, \quad \lim _{\Omega \rightarrow 0} e^{i J \phi}=e^{i \lambda^{\prime} \tilde{\phi}} .
$$

In principle, we could choose to contract the conformal algebra $\mathrm{su}(1,1)$ alone, i.e., not to touch the R symmetry sector at all. However, to have a well-defined contraction limit for the full superconformal algebra (including odd generators) requires that the contraction procedure must be the same for both algebras (see [13]).

The new variables $\tilde{\psi}, \tilde{\phi}$ run in the interval $-2 \pi / \Omega^{2} \leq \tilde{\psi}, \tilde{\phi}<2 \pi / \Omega^{2}$. Therefore, in the contraction limit they cease to be angular coordinates and become coordinates on $\mathbb{R}$. At the same time, the exponential functions (71) obtained in the contraction limit are naturally $\delta$-function normalized. This switching from Kronecker to $\delta$-function normalization is in fact the well-known procedure of passing from a Fourier series to a Fourier integral expansion and it allows $\lambda, \lambda^{\prime}$ to take arbitrary (non-integer) values. In fact, taking the limit in this way we convert $\mathrm{su}(1,1) \oplus \mathrm{su}(2)$ into two copies of the harmonic oscillator algebra $\mathrm{h}(2)$, for which $\lambda$ and $\lambda^{\prime}$ are two different central charges (See Section 4.2). Clearly, this algebra does not contain the Hamiltonian, i.e., the external automorphism U(1) generator. Moreover, if we take the same limit at the level of the superconformal algebra whose bosonic subalgebra is $\mathrm{su}(1,1) \oplus \mathrm{su}(2)$, we would find out that the corresponding contracted superalgebra is 
not that of the pp-wave limit [3]. Therefore, we consider the contraction possibility (71) as trivial.

The second option to make the phase factor in (70) converge is to compensate the growth of $\Delta$ by that of $J$. To achieve this we set

$$
\Delta+J=\frac{\lambda}{\Omega^{2}}, \quad \Delta-J=\mu, \quad \psi+\phi=2 \hat{\psi}, \quad \psi-\phi=2 \Omega^{2} \hat{\phi}
$$

and obtain

$$
\lim _{\Omega \rightarrow 0} e^{i(\Delta \psi-J \phi)}=e^{i(\mu \hat{\psi}+\lambda \hat{\phi})} .
$$

This is not yet the end of the story: Forcing $J$ to grow, we may have trouble with the normalization factor in (40), unless we rescale the complex coordinate $\tau$ on the sphere in exactly the same way as that on the disc (see eq. (65)), $\tau \rightarrow \Omega \tau$. Then the phase independent part of the basis vectors (40) has the well-defined limit

$$
\mathbf{g}_{n}=\frac{1}{\sqrt{n !}} \tau^{n} e^{-\frac{1}{2}|\tau|^{2}}
$$

(as before, the constant $\lambda$ has been absorbed into the coordinate). We recognize the basis for another copy of the Heisenberg algebra.

Combining all this, we finally obtain

$$
\lim _{\Omega \rightarrow 0} \Phi^{\Delta, J}(t, \bar{t}, \psi ; \tau, \bar{\tau}, \phi)=e^{i(\mu \hat{\psi}+\lambda \hat{\phi})} \sum_{m, n=0}^{\infty} c_{m n} t^{m} \tau^{n} e^{-\frac{1}{2}|t|^{2}-\frac{1}{2}|\tau|^{2}} .
$$

Note that according to Eq. (72) the new variables $\hat{\psi}$ and $\hat{\phi}$ run over the intervals $-2 \pi \leq \hat{\psi}<$ $2 \pi$ and $-2 \pi / \Omega^{2} \leq \hat{\phi}<2 \pi / \Omega^{2}$, respectively. Therefore, in the contraction limit $\hat{\phi}$ becomes a central charge coordinate, while $\hat{\psi}$ remains an angular $\mathrm{U}(1)$ coordinate. Thus, the effect of the limiting procedure (72) is to preserve the diagonal $\mathrm{u}(1)$ subalgebra in $\mathrm{su}(1,1) \oplus \mathrm{su}(2)$. Since this is what one expects from the contracted superconformal algebra in the pp-wave context (see [3, 13]), we call this limit non-trivial.

\subsection{The Heisenberg algebra $h(2)$ as a contraction of $\mathrm{su}(1,1) \oplus \mathrm{su}(2)$}

Let us now examine what happens to the algebra $\mathrm{su}(1,1) \oplus \mathrm{su}(2)$ in the trivial (71) or non-trivial (72) limits. Since in the case (71) the conformal and the R symmetry algebras are contracted independently, it is enough to consider the contraction of $\mathrm{su}(1,1)$. We now rescale $t$ and replace $\psi$ by $\tilde{\psi}$ not just in the fields, but directly in the generators (47) of the algebra $\mathrm{su}(1,1)$. It is clear that all the generators diverge, unless we introduce appropriate rescalings:

$$
\begin{aligned}
& \mathcal{Q}=\lim _{\Omega \rightarrow 0} \Omega^{2} M_{0}=-i \frac{\partial}{\partial \tilde{\psi}} \\
& \mathcal{M}_{+}=\lim _{\Omega \rightarrow 0} \Omega \sqrt{2} M_{+}=i \frac{\partial}{\partial t}-\frac{\bar{t}}{2} \frac{\partial}{\partial \tilde{\psi}} \\
& \mathcal{M}_{-}=\lim _{\Omega \rightarrow 0} \Omega \sqrt{2} M_{-}=i \frac{\partial}{\partial \bar{t}}+\frac{t}{2} \frac{\partial}{\partial \tilde{\psi}}
\end{aligned}
$$


It is easy to check that these generators satisfy the following harmonic oscillator algebra

$$
\left[\mathcal{M}_{-}, \mathcal{M}_{+}\right]=\mathcal{Q}, \quad\left[\mathcal{Q}, \mathcal{M}_{ \pm}\right]=0
$$

where $\mathcal{Q}$ is the central element and $\mathcal{M}_{ \pm}$are the creation and annihilation operators. Note the absence of the Hamiltonian, i.e., of a $\mathrm{U}(1)$ generator rotating $\mathcal{M}_{ \pm}$.

The second limiting procedure (72) leads to the following generators:

$$
\begin{aligned}
& \mathcal{H}=\lim _{\Omega \rightarrow 0}\left(M_{0}+L_{0}\right)=-i \frac{\partial}{\partial \hat{\psi}} \quad \mathcal{Q}=\lim _{\Omega \rightarrow 0} \Omega^{2}\left(M_{0}-L_{0}\right)=-i \frac{\partial}{\partial \hat{\phi}} \\
& \mathcal{L}_{+}=\lim _{\Omega \rightarrow 0} \Omega \sqrt{2} L_{+}=e^{i \hat{\psi}}\left[i \frac{\partial}{\partial \tau}-\frac{\bar{\tau}}{2} \frac{\partial}{\partial \hat{\phi}}\right] \quad \mathcal{L}_{-}=\lim _{\Omega \rightarrow 0} \Omega \sqrt{2} L_{-}=e^{-i \hat{\psi}}\left[i \frac{\partial}{\partial \bar{\tau}}+\frac{\tau}{2} \frac{\partial}{\partial \hat{\phi}}\right] \\
& \mathcal{M}_{+}=\lim _{\Omega \rightarrow 0} \Omega \sqrt{2} M_{+}=e^{i \hat{\psi}}\left[i \frac{\partial}{\partial t}-\frac{\bar{t}}{2} \frac{\partial}{\partial \hat{\phi}}\right] \quad \mathcal{M}_{-}=\lim _{\Omega \rightarrow 0} \Omega \sqrt{2} M_{-}=e^{-i \hat{\psi}}\left[i \frac{\partial}{\partial \bar{t}}+\frac{t}{2} \frac{\partial}{\partial \hat{\phi}}\right]
\end{aligned}
$$

and algebra:

$$
\begin{aligned}
& {\left[\mathcal{M}_{-}, \mathcal{M}_{+}\right]=\mathcal{Q}, \quad\left[\mathcal{L}_{-}, \mathcal{L}_{+}\right]=\mathcal{Q}} \\
& {\left[\mathcal{H}, \mathcal{M}_{ \pm}\right]= \pm \mathcal{M}_{ \pm}, \quad\left[\mathcal{H}, \mathcal{L}_{ \pm}\right]= \pm \mathcal{L}_{ \pm}}
\end{aligned}
$$

Clearly, $\mathcal{M}_{ \pm}, \mathcal{L}_{ \pm}$, and $\mathcal{Q}$ generate the algebra $\mathrm{h}(2)$, i.e. two copies of the Heisenberg algebra. The two algebras share the same central element $\mathcal{Q}$ and external $\mathrm{U}(1)$ automorphism $\mathcal{H}$. Then it becomes clear that the charge $\lambda$ of the conformal field (75) associated with the coordinate $\hat{\phi}$ corresponds to the central charge $\mathcal{Q}$ in (79), while the charge $\mu$ associated with the coordinate $\hat{\psi}$ corresponds to the $\mathrm{U}(1)$ automorphism generator $\mathcal{H}$. In physics $\mathcal{H}$ is interpreted as the Hamiltonian $1 / 2\left(p^{2}+q^{2}\right)$. The difference $\mu=\Delta-J$ can be viewed as the anomalous dimension of a CFT operator with canonical dimension $\Delta_{0}=J$. In the dual string theory the charge $\lambda$ is identified with the light-cone momentum $p_{+}$of a free string moving in the pp-wave background [6].

Let us summarize what we have done. We started from the simple geometric postulate of expanding the unit disc, on which the one-dimensional conformal fields live. To make the basis vectors for such fields survive, we had to let their conformal dimension $\Delta$ grow like the disc radius squared. Then, to compensate the divergence in the $\mathrm{U}(1)$ factor which carries the UIR weight we had either to transform this U(1) charge into a central charge, or to seek help from the R symmetry sector by combining two $\mathrm{U}(1)$ charges into one central charge. In the latter case it was important that in both the conformal and $\mathrm{R}$ symmetry sectors we could find similar U(1) factors. This explains why we had to use a realization of the conformal fields with manifest action of $\mathrm{SO}(2)$ rather than the more familiar dilatation $\mathrm{SO}(1,1)$. As a result, we found a generalized Inönü-Wigner contraction of the algebra $\mathrm{su}(1,1) \oplus \mathrm{su}(2)$. This contraction has been performed, at the abstract algebraic level, in the four-dimensional case $\mathrm{su}(2,2) \oplus \mathrm{su}(4) \sim \mathrm{so}(4,2) \oplus \mathrm{so}(6)$ in Ref. 13] (we quote this result in Appendix B). There it has also been shown that this type of contracted (super)algebras underlie the "pp-wave limit" proposal of Ref. [2].

Finally, we remark that in the four-dimensional case the corresponding contraction procedure would lead to the Fock-Bargmann realization of the Heisenberg algebra $\mathrm{h}(8)$ on 
functions over the complex space $\mathbb{C}^{8}$. This representation is unitary equivalent, via the integral transform from the Fock-Bargmann picture to the familiar (coordinate) Schrödinger representation of $\mathrm{h}(8)$ acting on functions over the real space $\mathbb{R}^{8}$, the latter being the target

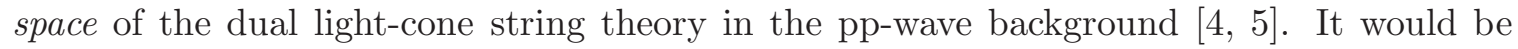
interesting to understand better the relationship between the CFT and the corresponding dual supergravity (string) theory from this point of view.

\subsection{Contraction of Minkowski fields}

One may wonder if it is possible to carry out the contraction procedure directly for the conformal fields defined in the compactified Minkowski space $\overline{\mathbb{M}}$. In our simplified situation $\overline{\mathbb{M}}^{1}=S^{1}$. It is known that the discrete series $\mathcal{D}_{\ell}^{ \pm}$of $\mathrm{SU}(1,1)$ can be realized in the space of functions on the unit circle as well (see, e.g., Ref. [14]). In particular, the functions of $\mathcal{D}_{\ell}^{+}$ are expanded in a Fourier series,

$$
f(\theta)=\sum_{n=0}^{\infty} a_{n} e^{i n \theta}
$$

and may be viewed as limiting (boundary) values of functions holomorphic inside the unit disc (cf. (53)). The action of the infinitesimal generators (3) is given by

$$
\begin{aligned}
& M_{+}=-i e^{-i \theta} d / d \theta \\
& M_{-}=i e^{-i \theta} d / d \theta-2 \ell e^{i \theta} \\
& M_{0}=\ell+i d / d \theta .
\end{aligned}
$$

Note that in (80) the conformal field is expanded over the basis $e^{i n \theta}$ in which the compact $\mathrm{SO}(2)$ generator $M_{0}$ is diagonal.

In Section 4.2 we explained how to perform the pp-wave contraction at the level of the Lie algebra. Now we have a different explicit realization of the same algebra. Requiring the generators (81) to stay non-singular in the contraction limit, we have to try to find the corresponding contraction prescription for the single coordinate of the carrier space $\theta$. Considering $\theta$ as a function of $\Omega$ and demanding, for instance, $\Omega^{2} M_{0}$ to be finite when $\Omega \rightarrow 0$, we deduce that either $\ell \sim 1 / \Omega^{2}$ and $\theta \sim\left(a_{1}+a_{2} \Omega+a_{3} \Omega^{2}\right)$ or $\ell \sim 1 /\left(a_{1}+a_{2} \Omega+a_{3} \Omega^{2}\right)$ and $\theta \sim \Omega^{2}$, where one of the coefficients $a_{i}$ can be non-zero. In neither of these two cases a non-singular limit for the generators $\Omega M_{ \pm}$can be obtained. In the case of the expanding unit disc we had an additional radial coordinate whose expansion rate was adjusted to compensate the contraction of the Lie algebra generators. In the present situation the expansion of the unique angular coordinate is not sufficient to compensate the different contraction rates of the generators $M_{ \pm}$and $M_{0}$. Thus, we are lead to the conclusion that a smooth pp-wave contraction limit for conformal fields defined in the compactified Minkowski space is not possible.

Finally, let us recall the alternative realization of the conformal fields in Minkowski space viewed as a coset of the group $\operatorname{SL}(2, \mathbb{R})$ (Section 3.4). The distinctive feature of this realization is that the fields transform homogeneously under the non-compact dilatation group $\mathrm{SO}(1,1)$ instead of the compact $\mathrm{SO}(2)$. This is reflected in the exponential factor $e^{-\ell a}=e^{\Delta a}$ in eq. (64). Trying to perform the contraction on such fields, we have no choice but to rescale the $\mathrm{SO}(1,1)$ carrier coordinate $a$ and thus to transform the weight $\Delta$ into 
a central charge. Indeed, the compact $\mathrm{R}$ symmetry group does not contain an $S O(1,1)$ subgroup, so it cannot provide a compensating factor for the diverging non-compact factor $e^{\Delta a}$. We are lead to the conclusion that when trying to perform the contraction not just at the abstract algebraic level, but on the UIRs of the algebra (i.e., on the fields) we have to carefully choose the space where these UIRs are defined.

\section{Correlation functions in the contraction limit}

The central point in CFT is the study of correlation functions. From the group-theoretical point of view, these are multi-point covariants of the conformal group. As we have seen, the pp-wave limit is essentially a transition from field theory to quantum mechanics, where "a second-quantized field" describing a multiparticle system degenerates into the wave function of "a first-quantized particle". Therefore, the very notion of a correlation function becomes rather obscure and the relevant question we may ask is: Can the multi-point covariants of the conformal and R symmetry groups survive in this quantum mechanical limit, where the conformal weight and the $\mathrm{R}$ charge become infinite?

Here we propose a possible answer to this question. 10

\subsection{Two-point functions}

Let us begin with the simplest case of the two-point functions. Consider first the conformal group $\mathrm{SU}(1,1)$ alone. A suitable two-point covariant can be constructed very easily, remembering our interpretation of the conformal fields as functions on $\mathrm{G}$, covariant under the left action of $\mathrm{K}$ but invariant under the right action of G. Let us take two copies $\mathbf{g}_{1}, \mathbf{g}_{2}$ of the $\mathrm{SU}(1,1)$ matrices (51) and denote their entries by $\mathbf{g}_{ \pm}{ }^{i}$ (see Section 3.3.1). Then it is clear that the two-point functions

$$
\left(\mathbf{1}_{ \pm} \mathbf{2}_{ \pm}\right) \equiv\left(\mathbf{g}_{1}\right)_{ \pm}{ }^{i} \epsilon_{i j}\left(\mathbf{g}_{2}\right)_{ \pm}^{j}
$$

are invariant under the right action of the group (i.e., the action on the indices $i, j$ ), and covariant under the left action (i.e., the action on the indices \pm ). In particular,

$$
\left(\mathbf{1}_{-} \mathbf{2}_{-}\right)=e^{-i\left(\psi_{1}+\psi_{2}\right) / 2} \frac{t_{1}-t_{2}}{\sqrt{\left(1-\left|t_{1}\right|^{2}\right)\left(1-\left|t_{2}\right|^{2}\right)}}
$$

is a two-point covariant carrying weight $\Delta=-\ell=-1 / 2$ at each point, while

$$
\left(\mathbf{1}_{-} \mathbf{2}_{+}\right)=e^{i\left(-\psi_{1}+\psi_{2}\right) / 2} \frac{t_{1} \bar{t}_{2}-1}{\sqrt{\left(1-\left|t_{1}\right|^{2}\right)\left(1-\left|t_{2}\right|^{2}\right)}}
$$

carries weight $\Delta_{1}=-1 / 2$ at point 1 and $\Delta_{2}=1 / 2$ at point $2 . \square$

\footnotetext{
${ }^{10}$ The discussion in this section is largely inspired by experience with harmonic superspace $18,19,28$, where one systematically uses coordinates for the $\mathrm{R}$ symmetry group on the same footing as the space-time coordinates.

${ }^{11}$ The modulus of expression (34) is the "invariant distance" between points 1 and 2 in the Riemannian sense.
} 
Two-point functions with equal positive weights $\Delta>0$ can be obtained as follows:

$$
\left\langle\Phi^{\Delta} \mid \Phi^{\Delta}\right\rangle_{S U(1,1)}=\frac{1}{\left(\mathbf{1}_{-} \mathbf{2}_{-}\right)^{2 \Delta}}=e^{i \Delta\left(\psi_{1}+\psi_{2}\right)} \frac{\left(1-\left|t_{1}\right|^{2}\right)^{\Delta}\left(1-\left|t_{2}\right|^{2}\right)^{\Delta}}{\left(t_{1}-t_{2}\right)^{2 \Delta}} .
$$

In accordance with our interpretation of the fields $\Phi^{\ell}$ as lowest weight vectors of the left algebra (55) (see (56)), we obtain

$$
\begin{aligned}
& M_{0}\left\langle\Phi^{\Delta} \mid \Phi^{\Delta}\right\rangle=\left\langle\Phi^{\Delta} \mid \Phi^{\Delta}\right\rangle \overleftarrow{M}_{0}=\Delta\left\langle\Phi^{\Delta} \mid \Phi^{\Delta}\right\rangle \\
& M_{-}\left\langle\Phi^{\Delta} \mid \Phi^{\Delta}\right\rangle=\left\langle\Phi^{\Delta} \mid \Phi^{\Delta}\right\rangle \overleftarrow{M}_{-}=0
\end{aligned}
$$

It should be stressed that when checking eq. (87) we have neglected the singularity at the coincident point $t_{1}=t_{2}$, which is a typical property of the two-point functions of physical fields (in reality the right-hand side of eq. (37) contains a contact term).

Note that there exists another right-invariant expression with the same weight $\Delta$, $\left(\mathbf{1}_{+} \mathbf{2}_{+}\right)^{2 \Delta}$. It is not a suitable two-point function, because it is annihilated by $M_{+}$instead of $M_{-}$, so it corresponds to a highest weight vector of positive weight. As a consequence, the explicit expression of $\left(\mathbf{1}_{+} \mathbf{2}_{+}\right)^{2 \Delta}$ contains the antiholomorphic polynomial $\left(\bar{t}_{1}-\bar{t}_{2}\right)^{2 \Delta}$. This means that we are dealing with a finite-dimensional non-unitary representation of $\mathrm{SU}(1,1)$.

The two-point covariant (84) is of a different nature. First of all, since the variables $t_{1,2}$ are restricted to the interior of the unit disc, $\left|t_{1,2}\right|<1$, this expression never vanishes. Then the two-point function

$$
\left\langle\Phi^{\Delta} \mid \bar{\Phi}^{-\Delta}\right\rangle_{S U(1,1)}=\frac{(-1)^{2 \Delta}}{\left(\mathbf{1}_{-} \mathbf{2}_{+}\right)^{2 \Delta}}=e^{i \Delta\left(\psi_{1}-\psi_{2}\right)} \frac{\left(1-\left|t_{1}\right|^{2}\right)^{\Delta}\left(1-\left|t_{2}\right|^{2}\right)^{\Delta}}{\left(1-t_{1} \bar{t}_{2}\right)^{2 \Delta}}
$$

is non-singular at the coincident point, unlike (85). Secondly, eq. (87) is replaced by

$$
M_{-}\left\langle\Phi^{\Delta} \mid \bar{\Phi}^{-\Delta}\right\rangle=\left\langle\Phi^{\Delta} \mid \bar{\Phi}^{-\Delta}\right\rangle \overleftarrow{M}_{+}=0
$$

This means that the two-point function (88) corresponds to a lowest-weight irrep (holomorphic) of positive weight $\Delta$ at point 1 and to a highest-weight irrep (antiholomorphic) of negative weight $-\Delta$ at point 2. In fact, this is the so-called Bergman kernel [29, 30] used to project the Hilbert space of functions square integrable inside the unite disc, onto holomorphic functions on the disc. Applied to a holomorphic field, this projector is the identity; in other words, (88) plays the rôle of a delta function for holomorphic fields:

$$
\Phi^{\Delta}(1)=\int_{2}\left\langle\Phi^{\Delta}(1) \mid \bar{\Phi}^{-\Delta}(2)\right\rangle_{S U(1,1)} \Phi^{\Delta}(2) .
$$

We remark that our two-point functions can also be regarded as functions on two copies of the homogenous space $\mathcal{D}^{1} \sim \mathrm{U}(1) \backslash \mathrm{SU}(1,1)$. To this end we should drop the phase factor in (85) or (88) (recall (53)) and examine the transformation properties of the resulting function of $t_{1}, t_{2}$ under the right group (generators (57)). Thus we make contact with the more familiar interpretation of the two-point functions. 


\subsection{Contraction of two-point functions}

Let us start with the non-singular two-point function (88). Using the prescriptions (65), (67) and (71), we easily obtain

$$
\lim _{\Omega \rightarrow 0}\left\langle\Phi^{\Delta} \mid \bar{\Phi}^{-\Delta}\right\rangle_{S U(1,1)}=e^{i \lambda\left(\tilde{\psi}_{1}-\tilde{\psi}_{2}\right)} e^{\lambda\left(t_{1} \bar{t}_{2}-\frac{1}{2}\left|t_{1}\right|^{2}-\frac{1}{2}\left|t_{2}\right|^{2}\right)} .
$$

It can be shown that after a transformation from the Fock-Bargmann to the Schrödinger (coordinate) realization of the Heisenberg algebra the expression (91) becomes just a delta function, in accord with the rôle of the function (88) before the contraction (recall (90)).

It is important to realize that the limit (91) has been obtained without the participation of the $\mathrm{R}$ symmetry sector. Consequently, the contracted two-point function carries no information about the "anomalous dimension" $\mu=\Delta-J$, the only quantum number of interest in the pp-wave limit. We note that this result corresponds to the two-point functions for the Heisenberg algebra proposed in [31].

Let us now turn to the singular two-point function (85). Trying to contract it, we run into two problems. Apart from the oscillating phase factor, we have to cope with the infinite power in $\left(t_{1}-t_{2}\right)^{-2 \Delta}$. As in Section 4.2, we can seek help from the R symmetry sector. Indeed, our fields are representations not only of the conformal group $\mathrm{SU}(1,1)$, but also of the R symmetry group $\mathrm{SU}(2)$. Thus, we have to construct two-point functions involving the $\mathrm{SU}(2)$ coordinates (39) as well. This can be done as above, by taking two SU(2) matrices $g_{1}, g_{2}$ and forming the right-invariant expression

$$
\left(1_{-} 2_{-}\right) \equiv\left(g_{1}\right)_{-}{ }^{i} \epsilon_{i j}\left(g_{2}\right)_{-}{ }^{j}=e^{-i\left(\phi_{1}+\phi_{2}\right) / 2} \frac{\tau_{1}-\tau_{2}}{\sqrt{\left(1+\left|\tau_{1}\right|^{2}\right)\left(1+\left|\tau_{2}\right|^{2}\right)}}
$$

which carries weight $J=\ell=1 / 2$ at both points. For arbitrary weight $J$ we can write down the two-point $\mathrm{SU}(2)$ covariant

$$
\langle J \mid J\rangle_{S U(2)}=\left(1_{-} 2_{-}\right)^{2 J}=e^{-i J\left(\phi_{1}+\phi_{2}\right)} \frac{\left(\tau_{1}-\tau_{2}\right)^{2 J}}{\left(1+\left|\tau_{1}\right|^{2}\right)^{J}\left(1+\left|\tau_{2}\right|^{2}\right)^{J}} .
$$

It satisfies equations similar to (86), (87). The main difference from the $\mathrm{SU}(1,1)$ case is that the two-point function (85) is singular at the coincident point, while (93) vanishes there. This is explained by the fact that $\mathrm{SU}(2)$ has finite-dimensional UIRs which are given by polynomials in the group coordinates $\tau$, see eq. (41).

The next step is to multiply the two expressions (85) and (93) to obtain the two-point function of fields with conformal weight $\Delta$ and isospin $J$ :

$$
\begin{aligned}
\left\langle\Phi^{\Delta, J} \mid \Phi^{\Delta, J}\right\rangle & =\frac{\Gamma(J+1)}{\Gamma(\Delta+1)} \frac{\left(1_{-} 2_{-}\right)^{2 J}}{\left(\mathbf{1}_{-} \mathbf{2}_{-}\right)^{2 \Delta}} \\
& =\frac{\Gamma(J+1)}{\Gamma(\Delta+1)} e^{i \Delta\left(\psi_{1}+\psi_{2}\right)-i J\left(\phi_{1}+\phi_{2}\right)} \frac{\left(1-\left|t_{1}\right|^{2}\right)^{\Delta}\left(1-\left|t_{2}\right|^{2}\right)^{\Delta}}{\left(1+\left|\tau_{1}\right|^{2}\right)^{J}\left(1+\left|\tau_{2}\right|^{2}\right)^{J}} \frac{\left(\tau_{1}-\tau_{2}\right)^{2 J}}{\left(t_{1}-t_{2}\right)^{2 \Delta}} .
\end{aligned}
$$

The choice of normalization is justified by the contraction limit below.

We already know that the phase factor is well behaved in the limit (72). However, the last factor in (94) is still divergent, unless we impose a condition relating the space-time and $\mathrm{R}$ symmetry variables, for instance,

$$
\left(t_{1}-t_{2}\right)^{2}=\left(\tau_{1}-\tau_{2}\right)^{2}
$$


or even stronger,

$$
t_{1}=\tau_{1} \quad \text { and } \quad t_{2}=\tau_{2} .
$$

This allows us to find the contraction limit

$$
\lim _{\Omega \rightarrow 0}\left\langle\Phi^{\Delta, J} \mid \Phi^{\Delta, J}\right\rangle=\left(\frac{2}{\lambda}\right)^{\mu} e^{i \mu\left(\hat{\psi}_{1}+\hat{\psi}_{2}\right)} e^{i \lambda\left[\left(\hat{\phi}_{1}+i\left|t_{1}\right|^{2}\right)+\left(\hat{\phi}_{2}+i\left|t_{2}\right|^{2}\right)\right]} \frac{1}{\left(t_{1}-t_{2}\right)^{2 \mu}} .
$$

We remark that before the contraction the coordinates $t$ and $\tau$ parametrize different spaces, the unit disc $\mathcal{D}^{1}$ and the two-sphere $S^{2}$, respectively. However, the former is a subspace of the latter, as $\mathcal{D}^{1}$ can be identified with, e.g., the southern hemisphere of $S^{2}$. In the contraction process the unit disc as well as the southern hemisphere expand and cover the whole complex plane. This allows us to perform the identification (96).

The meaning of eq. (96) is that the contracted two-point functions of the singular type can make sense only along certain hypersurfaces in the extended space $\mathbb{C}^{2}$ where the Heisenberg algebra $\mathrm{h}(2)(79)$ acts. In such a restricted subspace only half of the algebra $\mathrm{h}(2)$ is realized, as can be seen, e.g., by the fact that the function (97) is annihilated only by the sum of the left "annihilators" $\mathcal{L}_{-}+\mathcal{M}_{-}$. Alternatively, introducing new coordinates

$q_{ \pm}=1 / 2(t \pm \tau)$ as well as new generators $\mathcal{A}_{ \pm}=1 / \sqrt{2}\left(\mathcal{L}_{ \pm}+\mathcal{M}_{ \pm}\right)$, one can check that $\mathcal{A}_{ \pm}$ together with $\mathcal{Q}$ and $\mathcal{H}$ from eq. (78) obey the commutation relations of the algebra $\mathrm{h}(1)$ on the space of functions independent of the coordinate $q_{-}$.

It is important to realize that in the contraction limit of the singular two-point function the information about the anomalous dimension $\mu$ is not lost, it is explicitly present in the coordinate factor $1 /\left(t_{1}-t_{2}\right)^{2 \mu}$. This, in our opinion, makes meaningful the computation of the anomalous dimension in the pp-wave limit by QFT methods.

\subsection{Three- and four-point functions}

Once we have defined the two-point functions, we can easily construct multi-point functions as well. The correlation function of three fields $\Phi^{\Delta_{i}, J_{i}}\left(t_{i}, \tau_{i}\right), i=1,2,3$ is obtained by simply multiplying the two-point ones:

$$
\langle 1|2| 3\rangle=C_{123} \prod_{i=1}^{3} \frac{\Gamma\left(\alpha_{i} / 2+1\right)}{\Gamma\left(\beta_{i} / 2+1\right)} \frac{(12)^{\alpha_{1}}(23)^{\alpha_{2}}(31)^{\alpha_{3}}}{(\mathbf{1 2})^{\beta_{1}}(\mathbf{2 3})^{\beta_{2}}(\mathbf{3 1})^{\beta_{3}}},
$$

where

$$
\alpha_{i}=J_{i}-J_{j}-J_{k}, \quad \beta_{i}=\Delta_{i}-\Delta_{j}-\Delta_{k}, \quad i \neq j \neq k
$$

and we have used the short-hand notation $(12) \equiv\left(1_{-} 2_{-}\right)$, etc. The normalization factors $\Gamma\left(\alpha_{i} / 2+1\right) / \Gamma\left(\beta_{i} / 2+1\right)$ are needed for convergence in the contraction limit. The finite normalization $C_{123}$ is to be determined by the dynamics. The contraction can be performed after identifying the space-time and $\mathrm{R}$ symmetry coordinates at each point (recall (96)). The resulting expression contains three central charges $\lambda_{i}$ and anomalous dimensions $\mu_{i}$.

In the four-point case, for simplicity we consider four identical fields $\Phi^{\Delta, J}$. The most general four-point function then is

$$
\langle 1|2| 3 \mid 4\rangle_{\Delta, J}=\frac{\Gamma^{2}(J+1)}{\Gamma^{2}(\Delta+1)} \frac{[(12)(34)]^{2 J}}{[(\mathbf{1 2})(\mathbf{3 4})]^{2 \Delta}} \sum_{n=0}^{2 J} U^{n} f_{n}(u) .
$$


Here the covariant prefactor is a product of two-point functions with the corresponding weights. In addition, we have introduced a dependence on the conformal and R symmetry invariants ("cross-ratios") $u, U$. In our one-dimensional case there is only one such fourpoint invariant of each kind, which is easily obtained [28] by combining two-point covariants of the type (83), e.g.,

$$
u=\frac{(\mathbf{1 3})(\mathbf{2 4})}{(\mathbf{1 2})(\mathbf{3 4})} \quad \text { and } \quad U=\frac{(13)(24)}{(12)(34)}
$$

Note that the alternative choice $(\mathbf{1 4})(\mathbf{2 3})$ for the numerator is equivalent due to the cycling identity

$$
(12)(34)+(13)(42)+(14)(23)=0 .
$$

The reason why the dependence on $U$ in eq. (100) must be polynomial of order not greater than $2 J$ is that the fields $\Phi^{\Delta, J}$ belong to a finite-dimensional UIR of SU(2). Therefore the dependence on the $\mathrm{R}$ symmetry coordinates $\tau_{i}$ at each point must be polynomial (recall (41)). In other words, the singularity coming from the denominator in $U^{n}$ should not exceed the power of $(12)(34)$ in the prefactor in eq. (100). The dependence on the conformal cross-ratio $u$ is virtually arbitrary.

Finally, the contraction limit in (100) can be taken as before. The prefactor is wellbehaved owing to the $\Gamma$ factors and to the identification of the two spaces. The cross-ratios themselves are non-singular in the limit $\Omega \rightarrow 0$. We stress that in this limit the polynomial expansion in $U$ in (100) becomes an infinite power series. This is clearly due to the fact that the tensor product of two R symmetry irreps with infinite $J$ contains an infinite number of terms. At the same time, because of the identification of the space-time and $\mathrm{R}$ symmetry coordinates, the resulting four-point correlator will depend on an arbitrary function of a single cross-ratio. This function should, in principle, be determined by the dynamics of the theory.

\section{Acknowledgements}

We are grateful to V. Dobrev, E. Ivanov, S. Ferrara, S. Frolov, E. Kiritsis, S. Kuzenko, V. Petkova, R. Stora and S. Theisen for many useful discussions. G. A. was supported by the DFG and by the European Commission RTN programme HPRN-CT-2000-00131, and in part by RFBI grant N99-01-00166.

\section{Appendix A}

Here we find the orthogonal basis for conformal fields with manifest action of the dilatations, mentioned in Section 3.4. We adapt the discussion of Section 3.3 with some important modifications. First of all, since we are dealing with real matrices from $\operatorname{SL}(2, \mathbb{R})$, we can replace the complex spinor $z_{1}, z_{2}$ by a real one, $\xi_{1}, \xi_{2} \in \mathbb{R}$. Next, we replace the representation basis (22) by

$$
\mathrm{SL}(2, \mathbb{R}): \quad|\ell, \mu\rangle=N(\mu) \xi_{1}^{\mu} \xi_{2}^{2 \ell-\mu},
$$


where $\mu \in \mathbb{C}$ (the reason will become clear in a moment). As before, the group $\operatorname{SL}(2, \mathbb{R})$ acts on the spinor $\xi_{1}, \xi_{2}$ by linear transformations (cf. (25)). For the particular choice (62) this gives

$$
T^{\ell}(g)|\ell, \mu\rangle=N(\mu) e^{a(\mu-\ell)} \xi_{1}^{2 \ell}(x+\xi)^{2 \ell-\mu},
$$

where $\xi=\xi_{2} / \xi_{1}$ and we have assumed that $\xi_{1} \neq 0$. Now, we have to expand the right-hand side in the basis (103). However, we cannot proceed as we did after eq. (37). The reason is that this time the variable $x$ is not restricted to a finite domain, unlike the unit disc variable $t,|t|<1$ from (51). Thus, in (104) we cannot achieve, e,g., $|x / \xi|<1$ keeping $x$ and $\xi$ independent. This problem is a manifestation of the fundamental difference between coset spaces with compact and non-compact stability groups.

The correct expansion in the non-compact case is obtained through a Mellin transform (a generalization of the Fourier transform). We recall that a function $f(\xi), \xi>0$ such that $\xi^{c_{1}-1} f(\xi)$ and $\xi^{-c_{2}-1} f(\xi)$ are integrable for some $c_{1}, c_{2}>0$, admits the integral transform

$$
F(\lambda)=\int_{0}^{\infty} f(\xi) \xi^{\lambda-1} d \xi
$$

with inverse

$$
f(\xi)=\frac{1}{2 \pi i} \int_{c-i \infty}^{c+i \infty} F(\lambda) \xi^{-\lambda} d \lambda
$$

for any $-c_{2}<c<c_{1}$. So, the matrix elements in the basis (103) will be determined by the integral expansion

$$
T^{\ell}(g)|\ell, \mu\rangle=\frac{1}{2 \pi i} \int_{c-i \infty}^{c+i \infty} t^{\ell}(\mu, \nu ; g)|\ell, \nu\rangle d \nu
$$

Let us now apply the Mellin transform (105) to the factor $(x+\xi)^{2 \ell-\mu}$ in the right-hand side of eq. (104) (for $\xi>0$ ):

$$
F(\lambda)=\int_{0}^{\infty}(x+\xi)^{2 \ell-\mu} \xi^{\lambda-1} d \xi
$$

Restricting the coordinate $x$ to positive values only, we obtain

$$
F(\lambda)=x_{+}^{2 \ell-\mu+\lambda} \int_{0}^{\infty}(\rho+1)^{2 \ell-\mu} \rho^{\lambda-1} d \rho=\frac{\Gamma(\lambda) \Gamma(-2 \ell+\mu-\lambda)}{\Gamma(\mu-2 \ell)} x_{+}^{2 \ell-\mu+\lambda},
$$

where $\rho=\xi / x$ and the notation $x_{+}$is a reminder that this expansion is valid only for $x>0 . \mathbb{2}$ The convergence conditions in eq. (109) are

$$
0<\Re(\lambda)<\Re(\mu-2 \ell) .
$$

Finally, setting $\lambda=\nu-2 \ell$ in eq. (109) and comparing it with eqs. (103), (104) and (107), we find the matrix elements ${ }^{\mathrm{T}}$

$$
t_{+}^{\ell}(\mu, \nu ; a, x)=\frac{N(\mu)}{N(\nu)} \frac{\Gamma(\nu-2 \ell) \Gamma(\mu-\nu)}{\Gamma(\mu-2 \ell)} e^{a(\mu-\ell)} x_{+}^{\nu-\mu}, \quad 2 \ell<\Re(\nu)<\Re(\mu) .
$$

\footnotetext{
${ }^{12}$ The case $x<0$ can be treated similarly.

${ }^{13}$ We do need the explicit form of the normalization coefficients $N(\mu)$.
} 
So, the relevant basis vectors for expanding conformal fields of weight $\ell$ in a manifestly dilatation covariant way are the functions

$$
t_{+}^{\ell}(0, \nu ; a, x)=C(\nu) e^{-a \ell} x_{+}^{\nu}, \quad 2 \ell<\Re(\nu)<0
$$

(recall that $\ell<0$ for UIRs from the discrete series), and their analogs $t_{-}^{\ell}(0, \nu ; a, x)$ for $x<0$. They form an orthogonal set, e.g.

$$
\int_{0}^{\infty} \overline{x_{+}^{\nu^{\prime}}} x_{+}^{\nu} d x=2 \pi i \delta\left(\nu+\overline{\nu^{\prime}}+1\right)
$$

(this property is used to inverse the Mellin transform).

\section{Appendix B}

The bosonic part of the symmetry group of the $\mathcal{N}=4 \mathrm{SYM}$ is $\mathrm{SU}(2,2) \times \mathrm{SU}(4)$, which is locally isomorphic to $\mathrm{SO}(4,2) \times \mathrm{SO}(6)$. In the pp-wave limit this group (the Lie algebra) undergoes a generalized Inönü-Wigner contraction. It is useful to recall here the contraction procedure for the corresponding Lie algebra. Our presentation is similar to that of Ref. [13].

The Lie algebra so(4,2) with antisymmetric generators $M_{a b}, M_{a b}=-M_{b a}$, has the following commutation relations

$$
\left[M_{a b}, M_{c d}\right]=\eta_{b c} M_{a d}+\eta_{a d} M_{b c}-\eta_{a c} M_{b d}-\eta_{b d} M_{a c}
$$

where $a, b=0, \ldots, 5$ and the metric is $\eta=\operatorname{diag}(-,+, \ldots,+,-)$. It is useful to rewrite these relations by splitting the generators $M_{a b}$ as $\left(M_{0 i}, M_{i 5}, M_{i j}, M_{05}\right)$ with $i, j=1, \ldots, 4$ :

$$
\begin{array}{lll}
{\left[M_{0 i}, M_{0 j}\right]=M_{i j}} & {\left[M_{i 5}, M_{j 5}\right]=M_{i j}} \\
{\left[M_{0 i}, M_{j 5}\right]=\delta_{i j} M_{05}} & {\left[M_{i 5}, M_{k l}\right]=\delta_{i k} M_{l 5}-\delta_{i l} M_{k 5}} \\
{\left[M_{0 i}, M_{k l}\right]=\delta_{i k} M_{0 l}-\delta_{i l} M_{0 k}} &
\end{array}
$$

and

$$
\begin{aligned}
& {\left[M_{05}, M_{i 5}\right]=M_{0 i}} \\
& {\left[M_{05}, M_{0 i}\right]=-M_{i 5}} \\
& {\left[M_{05}, M_{k l}\right]=0}
\end{aligned}
$$

The generators $M_{i j}$ obey the commutation relations of the Lie algebra so(4). From (115) one concludes that the vectors $M_{0 i}$ and $M_{i 5}$ transform in the vector representation of so(4). The generator $M_{05}$ rotates $M_{i 5}$ into $M_{0 i}$ and $M_{0 i}$ into $-M_{i 5}$, so it is the generator of $\mathrm{SO}(2)$. It is worthwhile to note that since the algebra $\operatorname{so}(4,2)$ is defined over $\mathbb{R}$ one is not able to diagonalize $M_{05}$ in the vector space spanned by $M_{0 i}$ and $M_{i 5}$. ${ }^{\text {.4 }}$ Yet another convenient

\footnotetext{
${ }^{14}$ Recall that the generators of translations $P_{\mu}$, special conformal transformations $K_{\mu}$ and dilatations $D$ are expressed via $M_{a b}$ as $P_{\mu}=M_{\mu 5}-M_{\mu 4} K_{\mu}=M_{\mu 4}+M_{\mu 5}$ and $D=M_{54}$ with $\mu=0, . ., 3$. Thus, the $\mathrm{SO}(2)$ generator (usually referred to as the conformal Hamiltonian) is $M_{05}=\frac{1}{2}\left(P_{0}+K_{0}\right)$.
} 
set of generators is obtained by introducing $M_{0}=i M_{05}, M_{i}^{ \pm}=i / \sqrt{2}\left(M_{i 5} \pm i M_{0 i}\right)$. The corresponding commutation relations are

$$
\begin{aligned}
& {\left[M_{0}, M_{i}^{ \pm}\right]= \pm M_{i}^{ \pm}, \quad\left[M_{i}^{+}, M_{j}^{-}\right]=-\delta_{i j} M_{0}-M_{i j}} \\
& {\left[M_{i}^{ \pm}, M_{k l}\right]=\delta_{i k} M_{l}^{ \pm}-\delta_{i l} M_{k}^{ \pm} .}
\end{aligned}
$$

The Lie algebra so(6) with generators $L_{a b}, a, b=1, \ldots, 6$ has the same relations (114), except that the metric is now $\eta_{a b}=\delta_{a b}$. We rewrite the commutation relations by splitting the set of generators into $L_{i j}, L_{i 5}, L_{i 6}, L_{56}$ with $i, j=1, . ., 4$ :

$$
\begin{array}{rlrl}
{\left[L_{i 5}, L_{j 5}\right]} & =-L_{i j} & & {\left[L_{i 6}, L_{j 6}\right]=-L_{i j}} \\
{\left[L_{i 5}, L_{j 6}\right]} & =-\delta_{i j} L_{56} & & {\left[L_{i 6}, L_{k l}\right]=\delta_{i k} L_{l 6}-\delta_{i l} L_{k 6}} \\
{\left[L_{i 5}, L_{k l}\right]=\delta_{i k} L_{l 5}-\delta_{i l} L_{k 5},} & &
\end{array}
$$

and

$$
\begin{aligned}
& {\left[L_{56}, L_{i 5}\right]=-L_{i 6},} \\
& {\left[L_{56}, L_{i 6}\right]=L_{i 5},} \\
& {\left[L_{56}, L_{k l}\right]=0}
\end{aligned}
$$

Here $L_{i j}$ generates the subalgebra so(4) and $L_{56}$ is the generator of so(2). Like in the previous case, one can define $L_{0}=-i L_{56}$ and $L_{i}^{ \pm}=i / \sqrt{2}\left(L_{i 5} \pm i L_{i 6}\right)$ with commutation relations

$$
\begin{aligned}
& {\left[L_{0}, L_{i}^{ \pm}\right]= \pm L_{i}^{ \pm} \quad\left[L_{i}^{+}, L_{j}^{-}\right]=\delta_{i j} L_{0}+L_{i j}} \\
& {\left[L_{i}^{ \pm}, L_{k l}\right]=\delta_{i k} L_{l}^{ \pm}-\delta_{i l} L_{k}^{ \pm} .}
\end{aligned}
$$

To perform the pp-wave contraction one introduces a parameter $\Omega$ and rescales the generators as

$$
\mathcal{Q}=\Omega^{2}\left(M_{0}-L_{0}\right), \quad \mathcal{H}=M_{0}+L_{0}, \quad \mathcal{M}_{i}^{ \pm}=\Omega \sqrt{2} M_{i}^{ \pm}, \quad \mathcal{L}_{i}^{ \pm}=\Omega \sqrt{2} L_{i}^{ \pm},
$$

while the so(4) generators $L_{i j}=\mathcal{L}_{i j}$ and $M_{i j}=\mathcal{M}_{i j}$ are kept intact. The commutation relations for the new set of generators become $\Omega$-dependent and in the limit $\Omega \rightarrow 0$ one finds the algebra

$$
\begin{aligned}
& {\left[\mathcal{M}_{i}^{-}, \mathcal{M}_{j}^{+}\right]=\delta_{i j} \mathcal{Q}, \quad\left[\mathcal{L}_{i}^{-}, \mathcal{L}_{j}^{+}\right]=\delta_{i j} \mathcal{Q}, \quad\left[\mathcal{H}, \mathcal{M}_{i}^{ \pm}\right]= \pm \mathcal{M}_{i}^{ \pm}, \quad\left[\mathcal{H}, \mathcal{L}_{i}^{ \pm}\right]= \pm \mathcal{L}_{i}^{ \pm}} \\
& {\left[\mathcal{M}_{i}^{ \pm}, \mathcal{M}_{k l}\right]=\delta_{i k} \mathcal{M}_{l}^{ \pm}-\delta_{i l} \mathcal{M}_{k}^{ \pm}, \quad\left[\mathcal{L}_{i}^{ \pm}, \mathcal{L}_{k l}\right]=\delta_{i k} \mathcal{L}_{l}^{ \pm}-\delta_{i l} \mathcal{L}_{k}^{ \pm}}
\end{aligned}
$$

with $\mathcal{Q}$ being a central element. Clearly, $\mathcal{M}_{i}^{ \pm}, \mathcal{L}_{i}^{ \pm}$generate the algebra $\mathrm{h}(8)$, i.e. eight copies of the Heisenberg algebra with the common central element $\mathcal{Q}$, while $\mathcal{H}, \mathcal{M}_{i j}, \mathcal{L}_{i j}$ generate its external automorphism algebra $\mathrm{so}(2) \oplus \mathrm{so}(4) \oplus \mathrm{so}(4)$.

\footnotetext{
${ }^{15}$ The generators have the Hermitian conjugation property $L_{0}^{\dagger}=L_{0},\left(L^{ \pm}\right)^{\dagger}=L^{\mp}$, and similarly for $M_{0}, M^{ \pm}$.
} 


\section{References}

[1] O. Aharony, S. S. Gubser, J. M. Maldacena, H. Ooguri and Y. Oz, "Large N field theories, string theory and gravity," Phys. Rept. 323 (2000) 183, hep-th/9905111.

[2] M. Blau, J. Figueroa-O'Farrill, C. Hull and G. Papadopoulos, "A new maximally supersymmetric background of IIB superstring theory," JHEP 0201 (2002) 047, hepth/0110242.

[3] M. Blau, J. Figueroa-O'Farrill, C. Hull and G. Papadopoulos, "Penrose limits and maximal supersymmetry," Class. Quant. Grav. 19 (2002) L87, hep-th/0201081.

[4] R. R. Metsaev, "Type IIB Green-Schwarz superstring in plane wave Ramond-Ramond background," Nucl. Phys. B 625 (2002) 70, hep-th/0112044.

[5] R. R. Metsaev and A. A. Tseytlin, "Exactly solvable model of superstring in plane wave Ramond-Ramond background," hep-th/0202109.

[6] D. Berenstein, J. M. Maldacena and H. Nastase, "Strings in flat space and pp waves from N = 4 super Yang Mills," JHEP 0204 (2002) 013, hep-th/0202021.

[7] C. Kristjansen, J. Plefka, G. W. Semenoff and M. Staudacher, "A new double-scaling limit of $\mathrm{N}=4$ super Yang-Mills theory and PP-wave strings," hep-th/0205033.

[8] D. J. Gross, A. Mikhailov and R. Roiban, "Operators with large R charge in $\mathrm{N}=4$ Yang-Mills theory," hep-th/0205066.

[9] N. R. Constable, D. Z. Freedman, M. Headrick, S. Minwalla, L. Motl, A. Postnikov and W. Skiba, "PP-wave string interactions from perturbative Yang-Mills theory," hep-th/0205089.

[10] S. R. Das, C. Gomez and S. J. Rey, "Penrose limit, spontaneous symmetry breaking and holography in pp-wave background," hep-th/0203164.

[11] D. Berenstein and H. Nastase, "On lightcone string field theory from super Yang-Mills and holography," hep-th/0205048.

[12] R. G. Leigh, K. Okuyama and M. Rozali, "PP-waves and holography," hep-th/0204026.

[13] M. Hatsuda, K. Kamimura and M. Sakaguchi, "From super-AdS(5) x S**5 algebra to super-pp-wave algebra," hep-th/0202190.

[14] N. Ya. Vilenkin, Special Functions and Theory of Representations of Groups, Moscow, Nauka, 1965; Fonctions Speciales et Théorie des Représentations des Groupes, Paris, Dunod, 1969.

N. Ya. Vilenkin and A. U. Klimyk, Representations of Lie Groups and Special Functions, volume 1, Dordrecht/Boston/London, Kluwer Academic Publishers, 1993.

[15] A. Barut and C. Fronsdal, "On non-compact groups II. Representations Of The $2+1$ Lorentz Group," Proc. Roy. Soc. London A287 (1965) 532. 
[16] A. Knapp, Representation Theory of Semisimple Groups, Princeton University Press, Princeton, 1986.

[17] V.K. Dobrev, G. Mack, V. B. Petkova, S.G. Petrova and I.T. Todorov, Harmonic analysis, Lecture Notes in Physics, Springer-Verlag, 1977.

[18] A. S. Galperin, E. A. Ivanov, S. Kalitzin, V. I. Ogievetsky and E. S. Sokatchev, "Unconstrained $N=2$ matter, Yang-Mills and supergravity theories in harmonic superspace," Class. Quantum Grav. 1 (1984) 469. A. S. Galperin, E. A. Ivanov, V. I. Ogievetsky and E. S. Sokatchev, Harmonic Superspace, Cambridge, UK: Univ. Pr. (2001) 306 p.

[19] G. G. Hartwell and P. S. Howe, "(N, p, q) harmonic superspace," Int. J. Mod. Phys. A 10 (1995) 3901, hep-th/9412147; "A Superspace Survey," Class. Quant. Grav. 12 (1995) 1823.

[20] G. Mack and A. Salam, "Finite-component field representations of the conformal group," Ann. Phys. 53 (1969) 174.

[21] L. Van der Waerden, Gruppen Theoretische Methode in der Quantenmechanik, Berlin, Springer Verlag, 1932.

[22] E. Wigner, Group theory, New York, Academic Press, 1959.

[23] V. Bargmann, "Irreducible unitary representations of the Lorentz group," Ann. Math. 48 (1947) 568.

[24] G. Mack, "All unitary ray representations of the conformal group SU(2,2) with positive energy," Commun. Math. Phys. 55 (1977) 1.

[25] E. Fradkin and M. Palchik, Conformal quantum field theory in D-dimensions, Dordrecht/Boston/London, Kluwer Academic Publishers, 1996.

[26] I.T. Todorov, M.C. Mintchev and V.B. Petkova, Conformal Invariance in Quantum Field Theory, Pisa, Scuola Normale Superiore, 1978.

[27] E. Witten, "Coadjoint orbits of the Virasoro group," Comm. Math. Phys. 114 (1988) 1.

[28] B. U. Eden, P. S. Howe, A. Pickering, E. Sokatchev and P. C. West, "Four-point functions in $\mathrm{N}=2$ superconformal field theories," Nucl. Phys. B 581 (2000) 523, hep-th/0001138.

[29] Bergman S., "The kernel function and conformal mapping," A.M.S. Publ., 1950.

[30] W. Rühl, "Lectures on the Lorentz group and harmonic analysis", CERN Academic Training Programme, 1967-1968; Comm. Math. Phys. 30 (1973) 287.

[31] E. Kiritsis and B. Pioline, "Strings in homogeneous gravitational waves and null holography," hep-th/0204004. 